Article

\title{
Functional Analysis of the Fusion and Attachment Glycoproteins of Mojiang Henipavirus
}

\author{
Sofia Cheliout Da Silva ${ }^{1,2,+}$, Lianying Yan ${ }^{1,2}$, Ha V. Dang ${ }^{3} \mathbb{D}$, Kai Xu ${ }^{4}$, Jonathan H. Epstein ${ }^{5} \mathbb{D}$, David Veesler ${ }^{3}$ \\ and Christopher C. Broder 1,*(D)
}

1 Department of Microbiology, Uniformed Services University, Bethesda, MD 20814, USA; sofia.dasilva@hhs.gov (S.C.D.S.); lianying.yan.ctr@usuhs.edu (L.Y.)

2 Henry M. Jackson Foundation for the Advancement of Military Medicine, Bethesda, MD 20814, USA

3 Department of Biochemistry, University of Washington, Seattle, WA 98195, USA; hvdang@uw.edu (H.V.D.); dveesler@uw.edu (D.V.)

4 Vaccine Research Center, National Institutes of Allergy and Infectious Disease, National Institutes of Health, Bethesda, MD 20892, USA; xukai99@gmail.com

5 EcoHealth Alliance, New York, NY 10001, USA; epstein@ecohealthalliance.org

* Correspondence: christopher.broder@usuhs.edu; Tel.: +1-301-295-3401

+ Current Address: Biomedical Advanced Research and Development Authority, 200 C Street SW, Washington, DC 20215, USA.

check for updates

Citation: Cheliout Da Silva, S.; Yan, L.; Dang, H.V.; Xu, K.; Epstein, J.H.; Veesler, D.; Broder, C.C. Functional Analysis of the Fusion and Attachment Glycoproteins of Mojiang Henipavirus. Viruses 2021, 13, 517. https:// doi.org/10.3390/v13030517

Academic Editor: Heidi Drummer

Received: 1 January 2021

Accepted: 17 March 2021

Published: 22 March 2021

Publisher's Note: MDPI stays neutral with regard to jurisdictional claims in published maps and institutional affiliations.

Copyright: (c) 2021 by the authors. Licensee MDPI, Basel, Switzerland. This article is an open access article distributed under the terms and conditions of the Creative Commons Attribution (CC BY) license (https:// creativecommons.org/licenses/by/ $4.0 /)$.

\begin{abstract}
Mojiang virus (MojV) is the first henipavirus identified in a rodent and known only by sequence data, whereas all other henipaviruses have been isolated from bats (Hendra virus, Nipah virus, Cedar virus) or discovered by sequence data from material of bat origin (Ghana virus). EphrinB2 and -B3 are entry receptors for Hendra and Nipah viruses, but Cedar virus can utilize human ephrin-B1, -B2, -A2 and -A5 and mouse ephrin-A1. However, the entry receptor for MojV remains unknown, and its species tropism is not well characterized. Here, we utilized recombinant full-length and soluble forms of the MojV fusion (F) and attachment (G) glycoproteins in membrane fusion and receptor tropism studies. MojV F and G were functionally competent and mediated cell-cell fusion in primate and rattine cells, albeit with low levels and slow fusion kinetics. Although a relative instability of the pre-fusion conformation of a soluble form of MojV F was observed, MojV F displayed significantly greater fusion activity when heterotypically paired with Ghana virus G. An exhaustive investigation of A- and B-class ephrins indicated that none serve as a primary receptor for MojV. The MojV cell fusion phenotype is therefore likely the result of receptor restriction rather than functional defects in recombinant MojV F and G glycoproteins.
\end{abstract}

Keywords: mojiang virus; Cedar virus; paramyxoviridae; henipavirus; ephrin ligand; receptor tropism; envelope glycoprotein; nano luciferase; heptad repeat; membrane fusion

\section{Introduction}

Henipaviruses are unique members of the Paramyxoviridae family [1]. The prototypical henipavirus species, Hendra virus $(\mathrm{HeV})$ and Nipah virus $(\mathrm{NiV})$, are highly pathogenic Biological Safety Level-4 (BSL-4) select agents that emerged in the 1990s in Australia and peninsular Malaysia, respectively [2]. They possess a broad host range spanning six mammalian orders [3-5] and cause infections that can result in severe respiratory illnesses and/or encephalitis with associated high fatality rates in humans (40-100\%) [6,7] and other mammals, such as horses and pigs [3-5]. Both $\mathrm{HeV}$ and $\mathrm{NiV}$ periodically spill over from their bat reservoir hosts. NiV outbreaks are also associated with human-to-human infection transmission and have occurred in the Bangladesh/West Bengal region dubbed the "Nipah belt" on a near annual basis [8,9]. More recent instances of human NiV infection have occurred on the island of Mindanao in the Philippines in 2014 [10] and in the Malabar coast state of Kerala, India in 2018 [11]. However, although there is now a licensed vaccine 
against $\mathrm{HeV}$ infection for horses in Australia, no $\mathrm{NiV}$ and $\mathrm{HeV}$ therapeutics or vaccines approved for human use are currently available [12-14].

The genus Henipavirus also includes three additional species, two of which include viruses that were detected in or isolated from individual bats. The species, Ghanaian bat henipavirus, includes Ghana virus (GhV), which was identified by targeted RNA sequencing of fecal samples collected from straw-colored fruit bats (Eidolon helvum) [15]. Cedar virus (CedV) (Cedar henipavirus), a non-pathogenic virus, was isolated from fruit bat urine samples in Australia [16]. The third Henipavirus species, Mojiang virus (MojV) (Mojiang henipavirus), was discovered in 2012 specimens collected from yellow-breasted rats (Rattus flavipectus) in the Tongguan mine in Mojiang, Yunnan, China, where three miners had died of pneumonia of unknown etiology [17]. The clinical presentation of these infections and the confirmed exposure of the miners to horseshoe bats (genus Rinolophus), which are known to harbor SARS-like coronaviruses, have recently led to the suspicion, retrospectively, that these cases of pneumonia may be linked to SARS-like coronavirus infection [18]. No viral isolates of $\mathrm{GhV}$ and MojV have been recovered to date; $\mathrm{GhV}$ and MojV are known only from genetic sequence data $[17,19]$, and the pathogenic potential of either of these henipaviruses remains unknown.

Host cell infection by henipaviruses $\mathrm{HeV}, \mathrm{NiV}$ and $\mathrm{CedV}$ is mediated by their tetrameric attachment glycoprotein (G) and trimeric fusion glycoprotein (F) (reviewed in [20-22]). The G glycoprotein is expressed fully functional at the cell surface, whereas the F glycoprotein is initially expressed as an $\mathrm{F}_{0}$ inactive precursor, then recycled and cleaved by the endosomal protease cathepsin $\mathrm{L}$ to generate a biologically active protein composed of two subunits, $\mathrm{F}_{1}$ and $\mathrm{F}_{2}$, covalently linked by a disulfide bond and subsequently trafficked back to the plasma membrane [23]. Binding of the $G$ glycoprotein to an entry receptor triggers the $F$ glycoprotein to undergo an irreversible conformational change that mediates the merging of the virion and host cell membranes (reviewed in $[24,25]$ ). The F and G glycoproteinmediated membrane fusion is also responsible for the formation of multi-nucleated giant cells termed syncytia, a hallmark of cytopathic effects (CPE) observed in paramyxovirus infections [26].

Ephrin ligands, which engage in bidirectional signaling with Eph-receptors to mediate cell-cell repulsion, adhesion and attraction mechanisms involved in vascular and neural development, plasticity and repair (reviewed in $[27,28]$ ), have been identified as entry receptors of henipaviruses [29-31]. These ligands are conserved across mammalian species and classified into two groups: the A-class ephrins (A1 through A5) are tethered to the plasma membrane by a glycosylphosphatidylinositol (GPI) anchor, whereas the B-class ephrins (B1 through B3) are transmembrane proteins with a short cytoplasmic tail [28,32]. The ephrin-B2 and -B3 ligands act as entry receptors for $\mathrm{HeV}$ and $\mathrm{NiV}$ [33-35] through interactions between the binding cleft of the globular head of $\mathrm{G}$ and a specific domain of ephrin ligands termed the G-H loop $[29,32,36]$. CedV, however, is characterized by a uniquely broad ephrin tropism and can utilize mouse ephrin-A1, as well as human ephrinA2, -A5, -B1 and -B2 as entry receptors [31], whereas GhV G solely recognizes ephrin-B2 as its receptor $[30,37,38]$. MojV G, conversely, is unable to utilize the canonical ephrin-B2 or -B3 henipavirus receptors to mediate cell-cell fusion; moreover, MojV G does not bind the morbillivirus receptor CD150, nor does it bear sialic acid binding residues [39]. However, the ability of MojV F and G to mediate cell fusion by utilizing any other ephrin ligands is unknown. MojV species cell fusion tropism may be defined, at least in part, by receptor restrictions. Pteropid bat species are the natural reservoir of $\mathrm{HeV}, \mathrm{NiV}$, and $\mathrm{CedV}$ and Eidolon helvum is the putative reservoir for GhV [16,19,40,41]. Indeed, the ability of CedV $\mathrm{G}$ to functionally interact with multiple ephrins including murine ephrin-A1 [31], the fact that $\mathrm{MojV}$ is the first henipavirus identified in a rodent [17], and reports of productive experimental $\mathrm{HeV}$ and $\mathrm{NiV}$ infections in hamsters and guinea pigs [3-5], suggests that henipaviruses may naturally infect rodent hosts, perhaps through engagement with other ephrin ligands or rodent-specific ephrin ligands. 
In this study, we sought to characterize the functions of the MojV envelope glycoproteins, and we investigated MojV cell fusion species tropism and potential use of both A- and B-class ephrin ligands as fusion triggering receptors. We found that human, non-human primate and rodent cells are susceptible to cell-cell fusion mediated by MojV F and G glycoproteins with a low and slow fusion phenotype. This cell fusion phenotype was also observed with target cells expressing mouse ephrin-A1, rat ephrin-A4 and human ephrin-A5 ligands. Investigation of the basis for the MojV membrane fusion phenotype using an in vitro triggering assay with soluble MojV F (sF) revealed an instability of its pre-fusion conformation. However, a significantly greater cell fusion activity of MojV F was observed when heterotypically paired with GhV G. Taken together, these data indicate that the MojV cell fusion phenotype is likely the result of host cell receptor restrictions rather than inherent functional defects in recombinant MojV F and G.

\section{Materials and Methods}

\subsection{Cell Lines}

BHK-21 cells were obtained from Norman Cooper (National Institute of Health, Bethesda, MD, USA). BSR-T7/5 cells, BHK-21-derived cells that constitutively express T7 polymerase were provided by Tzanko Stantchev (U.S. Food and Drug Administration, Silver Spring, MD, USA). HeLa-USU cells lacking expression of $\mathrm{HeV}$ and $\mathrm{NiV}$ receptors ephrin-B2 and -B3 were described in previous studies [33]. HEK293T, A549 and Rat2 cells were provided by Brian Schaefer, Regina Day and Chou-Zen Giam (Uniformed Services University, Bethesda, MD, USA), respectively. Vero cells (ATCC CCL-81), CHO-K1 cells (ATCC CCL-61), Neuro-2a cells (ATCC CCL-131), C6 cells (ATCC CCL-107) and L2 cells (ATCC CCL-149) were purchased from the American Type Culture Collection (Manassas, VA, USA). FreeStyle ${ }^{\mathrm{TM}} 293$ cells were obtained from Thermo Fisher Scientific (Waltham, MA, USA). BHK-21, BSR-T7/5, Neuro-2a, HEK293T, HeLa-USU and Vero cells were cultured in Dulbecco's Modified Eagle's Medium (Quality Biologicals, Gaithersburg, MD, USA) with $2 \mathrm{mM}$ L-glutamine (Quality Biologicals,), 1\% penicillin-streptomycin (Quality Biologicals) and 10\% cosmic calf serum (HyClone, Logan, UT, USA) (DMEM-10). A549, CHO-K1 and L2 cells were maintained in Ham's F-12K (Kaighn's) Medium (Gibco, Gaithersburg, MD, USA) with $1 \%$ penicillin-streptomycin and $10 \%$ cosmic calf serum (F-12K-10). C6 cells were grown in Ham's F-12K (Kaighn's) Medium supplemented with 1\% penicillin-streptomycin, $2.5 \%$ cosmic calf serum and 15\% horse serum (ATCC 30-2040) (F-12K-2.5/15). FreeStyle ${ }^{\mathrm{TM}}$ 293 cells were maintained in Dulbecco's Modified Eagle's Medium supplemented with $2 \mathrm{mM}$ L-glutamine, $1 \%$ penicillin-streptomycin and 5\% cosmic calf serum (DMEM-5). All cell cultures were kept at $37^{\circ} \mathrm{C}$ in $5 \% \mathrm{CO}_{2}$ humidified atmosphere except for Neuro-2a cells and FreeStyle ${ }^{\mathrm{TM}} 293$ cells maintained in $8 \% \mathrm{CO}_{2}$.

\subsection{Expression Plasmids}

Codon optimized HeV (Genbank NC 001906.3), NiV (GenBank NC 002728.1), CedV (Genbank KP 271122.1), GhV (Genbank NC 025256.1) and MojV (Genbank NC 025352.1) F and $G$ open reading frames (ORFs) were synthesized by GenScript ${ }^{\circledR}$ (Piscataway, NJ, USA) and subcloned into the mammalian expression vector pcDNA3.1 Hygro CMV [42]. To facilitate detection an S peptide tag (S-tag) (KETAAAKFERQHMDS) was added to the end of the cytoplasmic domains of the full-length henipavirus glycoproteins by site-directed mutagenesis (QuickChange II XL, Agilent technologies, Santa Clara, CA, USA). For construction of soluble and secreted versions of F glycoproteins, the C-terminal cytoplasmic and transmembrane domains of CedV F and MojV F were replaced with a GCN4 trimerization motif (GCNt) [43] (MKQIEDKIEEILSKIYHIENEIARIKKLIGE), previously used in the generation of $\mathrm{sF}$ trimers of $\mathrm{HeV}$ and $\mathrm{NiV}$ [44,45], and an S-tag to generate trimeric soluble constructs of CedV sF and MojV sF (Figure S1). To produce tetrameric soluble GhV G (GhV sG) and MojV $\mathrm{G}(\mathrm{MojV} \mathrm{sG})$, the N-terminal cytoplasmic and transmembrane domains were replaced with an Igkleader sequence (METDTLLLWVLLLWVPGSTGD) and an S-tag followed by a GCN4 tetramerization motif (GCNtet) [43] (MKQIEDKLEEIESKLKKIENELARIKK), as previously 
described for CedV G (CedV sG) [31] (Figure S2). Expression plasmids encoding genes for human ephrin-A1, -A2, -A3, -A4, -A5, -B1, -B2 and -B3; mouse ephrin-A1; and rat ephrin-A4 were obtained from Origene ${ }^{\mathrm{TM}}$ (Rockville, MD, USA). S119P and P122S mutations were introduced in human and rat ephrin-A4, respectively, by site-directed mutagenesis. To create a soluble rat ephrin-A4, the region encoding L26-G171 was subcloned into the FC tag encoding plasmid BNN-CFC-pcDNA3.1. The Nano luciferase NLuc gene (Genbank LC 167158.1) was codon optimized and synthesized by GenScript ${ }^{\circledR}$ and subcloned under the control of the T7 promoter into a pTM1 provided by Chad Mire (University of Texas Medical Branch, Galveston, TX, USA) (pTM1-NLuc).

\subsection{Western Blot Analysis}

BSR-T7/5 cells grown to $60 \%$ confluency were transfected with S-tagged $\mathrm{HeV}$, CedV or MojV F or G constructs or with the empty vector using Lipofectamine ${ }^{\mathrm{TM}} \mathrm{LTX}$ (Invitrogen, Carlsbad, CA, USA) at a 1:2.1 ratio ( $\mu$ g DNA: $\mu$ L LTX). After $48 \mathrm{~h}$, lysates were collected in RIPA buffer (Thermo Fisher Scientific) with 1x cOmplete ${ }^{\mathrm{TM}}$ Protease Inhibitor Cocktail (Roche, Basel, Switzerland) and incubated with S protein agarose (EMD Biosciences Inc., Madison, WI, USA) at $4{ }^{\circ} \mathrm{C}$ overnight. Washed beads were boiled in reducing sample buffer (2x LDS NuPage ${ }^{\circledR}$ sample buffer (Invitrogen), 5\% $\beta$-mercaptoethanol (Sigma-Aldrich, St Louis, MO, USA)). Proteins were resolved by NuPage ${ }^{\circledR} 4-12 \%$ Bis-Tris SDS-PAGE (Invitrogen), and detected by Western blot with a horseradish peroxidase (HRP) conjugated anti-S polyclonal rabbit antibody $(1: 12,500)$ (Southern Biotechnology, Birmingham, AL, USA).

\subsection{Syncytium Formation Assay}

BSR-T7/5 and BHK-21 cells were grown to $60 \%$ confluency and transfected with pcDNA 3.1 Hygro CMV HeV, CedV or MojV F or G alone, or co-transfected with F and G $(1: 1)$ or empty vector, as described above. Cells were fixed in methanol and stained with crystal violet between $24 \mathrm{~h}$ and 5 days post-transfection. Brightfield microscope images were acquired with a Zeiss Axio Observer A1 inverted microscope (Carl Zeiss Microscopy, Jena, Germany) set to the $20 \times$ objective.

\subsection{Cell-Cell Fusion Nano Luciferase Reporter Gene Assay}

Effector BSR-T7/ 5 cells seeded at $0.6 \times 10^{5}$ cells per well (24-well tissue culture plates) were transfected $24 \mathrm{~h}$ after plating with $0.8 \mu \mathrm{g}$ total DNA. Cells were transfected with pcDNA 3.1 Hygro CMV HeV, CedV or MojV F or G alone, or co-transfected with homologous or heterologous F and G (1:1) HeV, NiV, CedV, GhV or MojV expression plasmid combinations or empty vector. Target HeLa-USU, A549, Vero, BHK-21, CHO-K1, L2, C6 and Rat 2 cells grown to $60 \%$ confluency were transfected with pTM1-NLuc $(1 \mu \mathrm{g}$ DNA:1 $\mu \mathrm{L}$ Plus ${ }^{\mathrm{TM}}$ Reagent:3 $\mu \mathrm{L}$ Lipofectamine ${ }^{\mathrm{TM}}$ LTX (Invitrogen)). NLuc was chosen as a reporter gene because the Nano luciferase enzyme is more stable and generates 150 -fold increased luminescence compared to firefly and renilla luciferases [46,47], allowing for more sensitive detection of low levels of cell-cell fusion. Target HEK293T cells were transfected with pTM1-NLuc using X-tremeGENE ${ }^{\mathrm{TM}} 9$ DNA Transfection Reagent (Roche, Basel, Switzerland) (1 $\mu \mathrm{g}$ DNA:3 $\mu \mathrm{L}$ X-tremeGENETM ${ }^{\mathrm{TM}}$. In the cell-cell fusion assays assessing ephrin ligand tropism, $\mathrm{CHO}-\mathrm{K} 1$ cells transfected with pCMV-human, mouse, or rat ephrin expression plasmids, in addition to pTM1-NLuc, were used as target cells instead. All target cells were trypsinized $24 \mathrm{~h}$ post-transfection and allowed to recover overnight. Target cells $\left(2.0 \times 10^{5}\right.$ cells per well) were then applied to the effector BSR-T7/5 cell monolayers. Effector/target cells mixtures were harvested 24 h, 48 h, 72 h, 96 h, 120 h, day 6 and day 7 post-application and assayed for luciferase activity in technical triplicates with the Nano-Glo ${ }^{\circledR}$ Luciferase Assay System (Promega, Madison, WI, USA). Data were acquired with a GloMax ${ }^{\circledR}$-Multi+ Detection System plate reader (Promega). Normalized maximum luminescence values (see data analysis) reached by each effector/target cell co-cultures are reported, and corresponding collection times are indicated in each figure. 


\subsection{Data Analysis}

Cell-cell fusion reporter gene assays were performed at least three times and in technical triplicates. Relative Luminescence Units (RLUs) from the various cell mixtures were normalized to RLUs from mixtures of each target cell type applied to effector cells transfected with the empty vector (background). Normalized RLUs were compared to background (value of 1) to assess statistical significance by applying Welch's $t$-test. Statistical analysis was performed with Graphpad Prism. Figures were prepared using Graphpad Prism and Adobe Photoshop.

\subsection{Protein Cross-Linking Assay}

BSR-T7/5 cells transiently expressing full length henipavirus glycoproteins were collected $72 \mathrm{~h}$ post-transfection and allowed to recover in DMEM- 10 overnight at $37^{\circ} \mathrm{C}$. Cells were washed and re-suspended in 1x PBS pH 7.4 (Quality Biologicals,). Aliquots of $5 \times 10^{6}$ cells were incubated with $0,1,3$ or $5 \mathrm{mM}$ BS3 (bis(sulfosuccinimidyl)suberate), No-Weigh ${ }^{\mathrm{TM}}$ Format (Thermo Fisher Scientific) for $45 \mathrm{~min}$ at $4{ }^{\circ} \mathrm{C}$. CedV and MojV sF and CedV, GhV and MojV sG purified proteins ( $1 \mu \mathrm{g}$ per sample) were incubated with 0, 0.8, 1.0 and $1.2 \mathrm{mM} \mathrm{BS} 3$ for $30 \mathrm{~min}$ at room temperature. All reactions were quenched for $15 \mathrm{~min}$ at room temperature with $20 \mathrm{mM}$ Tris. Full length proteins in cell lysates were precipitated with $\mathrm{S}$ agarose beads as described above. Cross-linked proteins were resolved by SDSPAGE NuPage ${ }^{\circledR} 4-12 \%$ Bis-Tris gels for F oligomers, 3-8\% Tris-Acetate gels (Invitrogen) for $\mathrm{G}$ oligomers and analyzed by Western blot.

\subsection{Production of Soluble Recombinant Proteins}

Previously described methods of production and purification of $\mathrm{HeV}$ and NiV sF proteins were applied to obtain the soluble henipavirus glycoproteins used in this study [45]. Briefly, all expression plasmids were transfected with Xtreme Fugene ${ }^{\circledR}{ }^{\circledR}$ (Roche, Basel, Switzerland) into FreeStyle ${ }^{\mathrm{TM}} 293$ cells with exception of the cell line expressing MojV sG which was established in Neuro-2a cells because of an unknown incompatibility of its full-length ectodomain expression in FreeStyle ${ }^{\mathrm{TM}} 293$ cells. To generate cell lines stably expressing the soluble proteins, transfected cells were subjected to drug selection in DMEM-5 containing $50 \mu \mathrm{g} / \mathrm{mL}$ Hygromycin B (Thermo Fisher Scientific) (DMEM-5+). After two rounds of limiting dilution cloning, candidate clones were confirmed by Western blot analysis and expanded in FreeStyle ${ }^{\mathrm{TM}} 293$ Expression Medium (Thermo Fisher Scientific). Clarified supernatants were filtered and subjected to affinity purification on an $S$ protein agarose affinity column (EMD Biosciences, San Diego, CA, USA). MojV sG (Figure S3A), MojV sF (Figure S3B), GhV sG (Figure S3C) and CedV sF (Figure S3D) glycoproteins were eluted, concentrated and purified before being subjected to size exclusion chromatography and analyzed by blue native (BN) PAGE and Western blotting as previously described [42,45]. MojV sG, CedV sG and GhV sG glycoproteins (Figure S2C) and the MojV sF and CedV sF glycoproteins (Figure S1C) were produced in their physiologically relevant oligomeric forms. Additional analysis by negative-staining electron microscopy (nsEM) of the quality of the previously undescribed purified MojV sG, and GhV sG glycoproteins (tetramers) and the MojV sF and CedV sF glycoproteins (trimers) is shown in Figure S4. A FreeStyle ${ }^{\mathrm{TM}} 293$ cell line stably expressing soluble rat ephrin-A4-Fc was created as described above with one modification; the supernatant was purified through a protein $\mathrm{G}$ agarose affinity column (EMD Biosciences) and eluted with a $0.1 \mathrm{M}$ Glycine pH 2.5 buffer.

\subsection{HR2 Peptide Triggering and Capture Assay}

Biotinylated peptides corresponding to the heptad repeat 2 (HR2) domain of CedV F (CedV FC2, KVDLSNEINKMNQSLKDSIFYLREAKRILDSVNISL) and MojV F (MojV FC2, KIDIGNQLAGINQTLQEAEDYIEKSEEFLKGVNPSI) were synthesized by New England Peptide $^{\mathrm{TM}}$, Inc. (Gardner, MA, USA) and are referred to as FC2 peptides in this study. A henipavirus F glycoprotein heptad-repeat capture assay was adapted from a previously 
established method [45,48]. Briefly, CedV sF or MojV sF glycoproteins were subjected to a combination of heat $\left(50^{\circ} \mathrm{C}\right)$, trypsin digestion $\left(4^{\circ} \mathrm{C}\right)$ and addition of the corresponding FC2peptide (1:6 ratio; sF:FC2 peptide) in different orders of treatment. The sF/FC2 peptide complexes were incubated for $1 \mathrm{~h}$ at $4{ }^{\circ} \mathrm{C}$ with a $50 \%$ avidin agarose slurry (Vector laboratories, Burlingame, CA, USA) in RIPA buffer and $1 \times$ cOmplete $^{\text {TM }}$ Protease Inhibitor Cocktail. Reduced samples were analyzed by Western blot with primary mouse anti-MojV F monoclonal antibody (mAb) 4G5 (1:2500), or anti-CedV F polyclonal serum (1:1000) and secondary HRP-conjugated goat anti-mouse $\operatorname{IgG}(\mathrm{H}+\mathrm{L})$ antibody $(1: 12,500)$ (Thermo Fisher Scientific).

\subsection{Generation of Antibodies against CedV and MojV sF Glycoproteins}

Mice studies were conducted in accordance with protocol MIC 16-262 approved by the Uniformed Service University Animal Care and Use Committee. Seven-week-old BALB/cByJ mice (stock\#001026) purchased from Jackson Labs (Bar Harbor, ME, USA) were immunized with purified MojV sF (Figure S3B) and CedV sF (Figure S3D) glycoproteins in sterile 1XPBS:MPL-TDM adjuvant and polyclonal and $\mathrm{mAbs}$ were produced as previously described [45]. The anti-MojV F mAb 4G5 is conformation independent.

\subsection{Sequence Analysis}

Sequences for human, mouse and rat ephrin ligands were aligned and analyzed with Clone Manager Suite9 software (Scientific \& Educational Software, Cary, NC, USA).

\subsection{Co-Precipitation Assay}

Soluble Fc-conjugated A-class and B-class ephrins including human ephrin-A1 Fc (provided by Kai $\mathrm{Xu}$, National Institutes of Health, Bethesda, MD, USA), mouse ephrin-A1, mouse ephrin-A2, human ephrin-A3, human ephrin-A4, human ephrin-A5, mouse ephrinB1, mouse ephrin-B2 and human ephrin-B3 (R\&D Systems; Minneapolis, MN, USA) and rat ephrin-A4 (see above) were incubated with purified MojV sG, GhV sG, and CedV sG proteins at a 1:2 ratio ( $\mu \mathrm{g}$ sG: $\mu \mathrm{g}$ ephrin) in RIPA buffer supplemented with $1 \mathrm{X}$ cOmplete protease inhibitor at $4{ }^{\circ} \mathrm{C}$ overnight. Samples were incubated with protein $\mathrm{G}$ agarose slurry or $\mathrm{S}$ protein agarose slurry for $2.5 \mathrm{~h}$ at $4{ }^{\circ} \mathrm{C}$. Co-precipitated henipavirus sG/ephrin ligand complexes were analyzed by Western blot as described above. Blots were probed with HRP-conjugated anti-S-tag rabbit polyclonal antibody.

\subsection{Biolayer Interferometry Assay}

Mouse ephrin-A1, human ephrin-A4, human ephrin-A5, human ephrin-B2 (R\&D Systems) and rat ephrin-A4 (see above) were diluted in kinetics buffer (KB: $1 \times$ PBS, $0.001 \%$ BSA, $0.02 \%$ Tween 20 and $0.005 \%$ NaN3 (ForteBio, Fremont, CA, USA)) to $600 \mathrm{nM}$. CedV sG was diluted to $14 \mu \mathrm{g} / \mathrm{mL}$ and GhV sG and MojV sG were diluted to $21 \mu \mathrm{g} / \mathrm{mL}$ in $10 \mathrm{mM}$ acetate buffer $\mathrm{pH}$ 5.0. Assays were performed as previously described [48] with immersion of CedV, GhV and MojV G-loaded AR2G sensors into the different ephrins solutions for 1000s (association phase) and then into KB for 1000s (dissociation phase).

\subsection{Electron Microscopic Imaging of Negative-Stained sG and sF}

Specimen preparation and electron microscopic imaging of negative-stained samples (nsEM) were performed following the conventional negative-staining protocol [49]. Briefly, $\mathrm{G}$ and $\mathrm{F}$ glycoproteins were diluted to a concentration of approximately $0.02 \mathrm{mg} / \mathrm{mL}$ with $10 \mathrm{mM}$ HEPES, pH 7.4, supplemented with $150 \mathrm{mM} \mathrm{NaCl}$. A 4.8- $\mu \mathrm{L}$ drop of the diluted sample was placed on a freshly glow-discharged carbon-coated copper grid. The drop was immediately removed with filter paper, and the grid was washed $3 x$ with the same buffer, followed by negative staining $3 \times$ with $0.75 \%$ uranyl formate. Datasets were collected using a Thermo Scientific Talos F200C transmission electron microscope operated at $200 \mathrm{kV}$ and equipped with a Ceta camera (Thermo Fisher Scientific). The nominal magnification was $57,000 \times$, corresponding to a pixel size of $2.53 \AA$, and the defocus was set at $-1.2 \mu \mathrm{m}$. 
Data were collected automatically using EPU. Single particle analysis was performed using CryoSPARC.

\section{Results}

\subsection{Expression of Recombinant Mojiang Virus Attachment and Fusion Glycoproteins}

MojV F and G glycoprotein expression constructs of codon optimized ORFs were synthesized and included an S-tag at the cytoplasmic end of each protein to facilitate detection (Figure 1A). Recombinant MojV F and G glycoproteins transiently expressed in BSRT7 / 5 cells displayed gel migration patterns similar to those of $\mathrm{HeV}$ and CedV F and G glycoproteins (Figure 1B) and were congruent with previous observations [39].

\section{A}

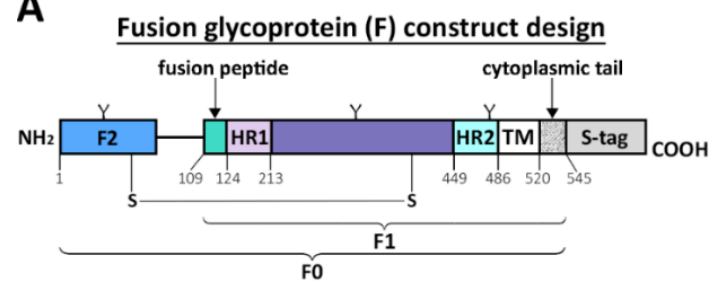

Attachment glycoprotein (G) construct design
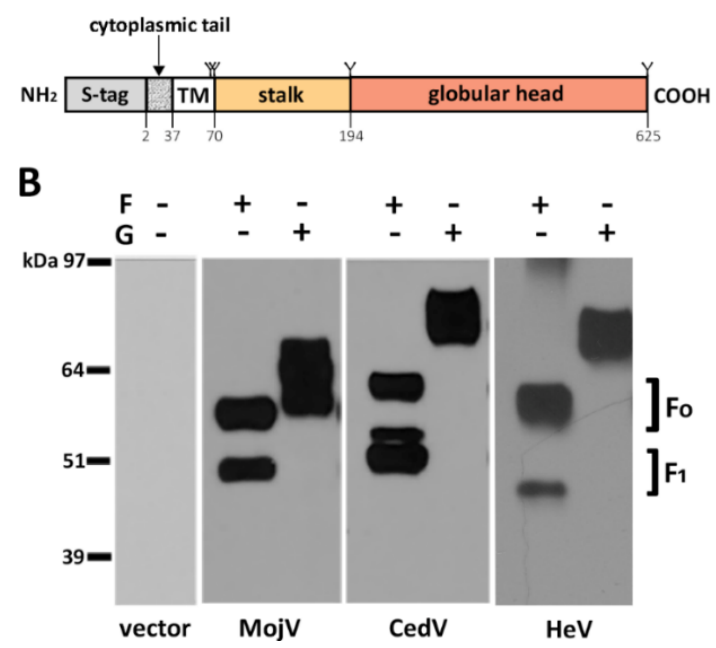

Figure 1. Recombinant Mojiang virus (MojV) fusion (F) and attachment (G) glycoprotein design and expression. (A) The open reading frames of MojV attachment $(\mathrm{G})$ and fusion $(\mathrm{F})$ glycoproteins were subcloned into a pcDNA3.1 Hygro CMV expression vector. The constructs were designed to express an S-peptide tag at the cytoplasmic tail end of each glycoprotein, at the C-terminus of $\mathrm{F}$ and at the $\mathrm{N}$-terminus of G. Functional domains of the attachment and fusion glycoproteins were predicted using Clustal Omega 1.2.4 sequence alignment [50], SWISS-MODEL homology modelling [51-55] and SABLE secondary structure prediction servers [56-58]. $\mathrm{F}_{0}$ : fusion glycoprotein precursor. $\mathrm{F}_{1}$, $\mathrm{F}_{2}$ : subunits of the mature fusion glycoprotein. TM: transmembrane domain. HR1: heptad repeat 1. HR2: heptad repeat 2. Y: N-glycosylation sites predicted by NetNGlyc 1.0 Server (Technical University of Denmark) at positions 69, 283 and 484 for MojV F and 61, 64, 189 and 619 for MojV G. The disulfide bond between C70 and C370 was predicted by the DISULFIND web server and is depicted as S-S [59]. (B) Transient expression of S-tagged MojV, CedV and HeV attachment and fusion glycoproteins in BSR-T7/5 cells. Cell lysates were precipitated with anti-S agarose beads $48 \mathrm{~h}$ post transfection. Western blot analysis was performed, and the membranes were probed with rabbit anti-S peptide:HRP antibodies (1:12,500).

The MojV, $\mathrm{HeV}$ and CedV F glycoproteins all displayed an $\mathrm{F}_{0} / \mathrm{F}_{1}$ band pattern indicating proper cellular proteolytic processing of the $F_{0}$ precursor into the mature $F_{1} / F_{2}$ form of the glycoproteins (Figure 1B). Densitometry analysis revealed that $\sim 50 \%$ of MojV $\mathrm{F}_{0}$, similar to $\mathrm{HeV}$ and $\mathrm{CedV} \mathrm{F}_{0}$, is processed into $\mathrm{F}_{1} / \mathrm{F}_{2}$ (Figure $1 \mathrm{~B}$ ). Because the $\mathrm{S}$-tag is 
located at the carboxyl-terminus of the $\mathrm{F}$ constructs, only the unprocessed $\mathrm{F}_{0}$ precursor and the $F_{1}$ subunit are detected. Analysis of MojV G by SDS-PAGE under reducing conditions revealed two major bands with apparent MWs of $\sim 62$ to $\sim 64 \mathrm{kDa}, \sim 10 \mathrm{kDa}$ smaller in comparison to HeV G $\sim 74$ to $76 \mathrm{kDa}$ [60] and CedV G $\sim 74$ to $76 \mathrm{kDa}$ (Figure 1B), likely due to the unusual absence of N-linked glycosylation sites in the $\beta$ propeller domain of its globular head as previously reported [39].

\subsection{Functional Assessment of Recombinant MojV F and G Glycoproteins}

The functionality of MojV F and G glycoproteins was qualitatively evaluated in a syncytium formation assay (Figure 2). While syncytia rapidly developed and spread through cell cultures co-expressing $\mathrm{HeV} \mathrm{F}$ and $\mathrm{G}$ or CedV F and $\mathrm{G}$ within $24 \mathrm{~h}$ posttransfection, syncytia in cells co-expressing MojV F and $\mathrm{G}$ were much less extensive and were only observed 5 days post-transfection (Figure 2A). The functionality of MojV F and $\mathrm{G}$ was confirmed by repeating this experiment in BHK-21 cells (Figure 2B). The kinetics of syncytia formation in BHK-21 cells co-transfected with MojV F and G differed from those observed in BSR-T7/5 and syncytia were detected 3 days post-co-transfection in BHK-21 cells (Figure 2B). In addition, syncytia formation mediated by MojV F and $G$ was not detected when this experiment was conducted using HEK293T or Vero cells (Figure S5A,B), which are known to be susceptible to $\mathrm{HeV}$, NiV [61] and CedV [31] F and G-mediated cell-cell fusion [39].

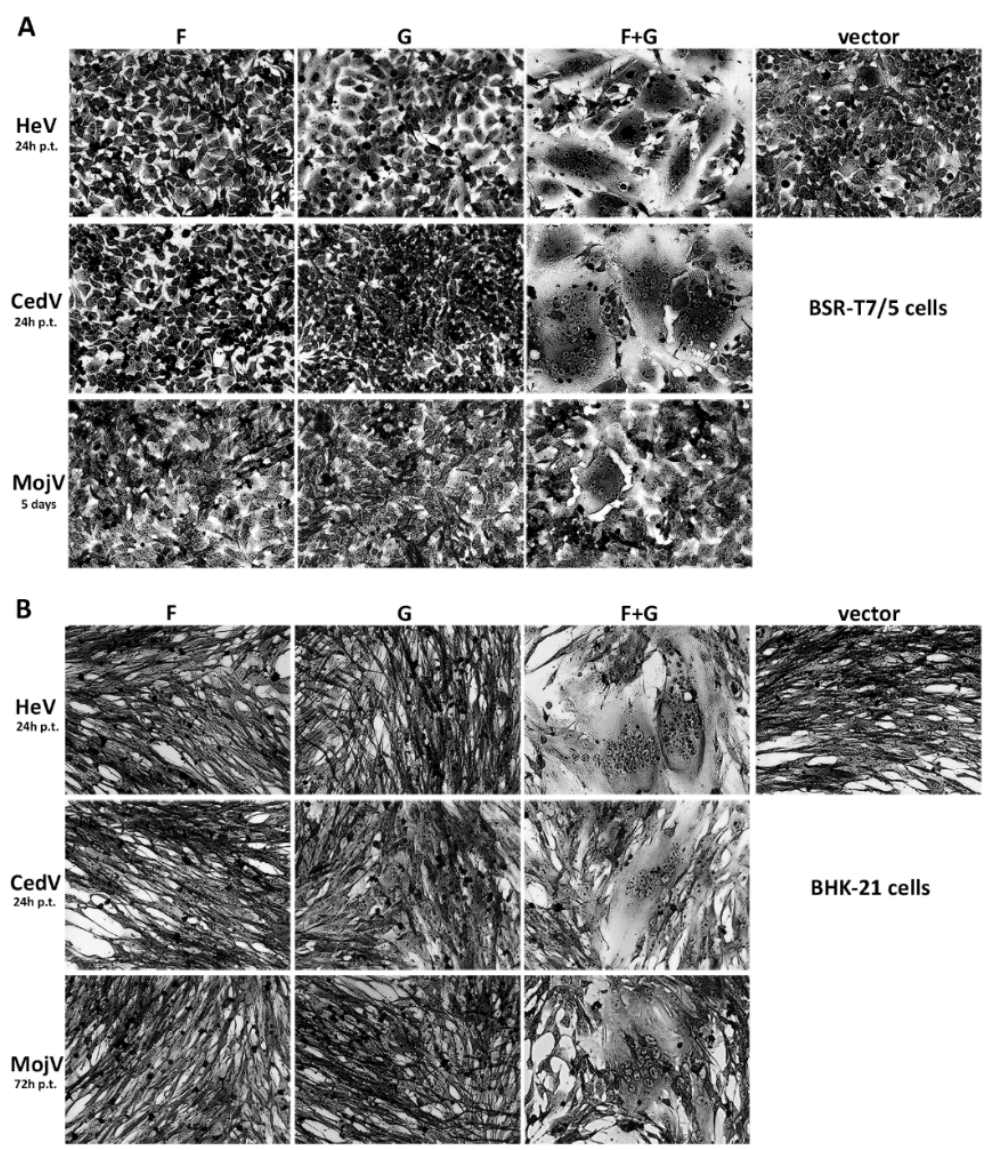

Figure 2. MojV F and $\mathrm{G}$ are functional and mediate cell-cell fusion as evidenced by syncytial formation. (A) BSR-T7/5 and (B) BHK-21 cells were transfected with pcDNA 3.1 Hygro CMV henipavirus $\mathrm{F}$ or $\mathrm{G}$ alone, or co-transfected with henipavirus $\mathrm{F}$ and $\mathrm{G}$ or with empty vector. Upon observation of giant multinucleated cell formations (syncytia), the cells were fixed in methanol and stained with crystal violet at the indicated time. Images were obtained with a Zeiss Axio Observer A1 inverted microscope with a $20 \times$ objective. p.t.: post-transfection. 


\subsection{Species Susceptibility to MojV F and G Mediated Fusion}

The susceptibility of mammalian cells to support MojV F and G-mediated membrane fusion was investigated quantitatively using human (HeLa-USU, A549, HEK293T), nonhuman primate (Vero) and rodent (BHK-21, CHO-K1, L2 rat, C6 rat, Rat2) cells as the target cell population with BSR-T7/5 cells expressing MojV F and G as effector cells or $\mathrm{HeV}$ or CedV F and $G$ as positive effector cell controls (Figure 3).

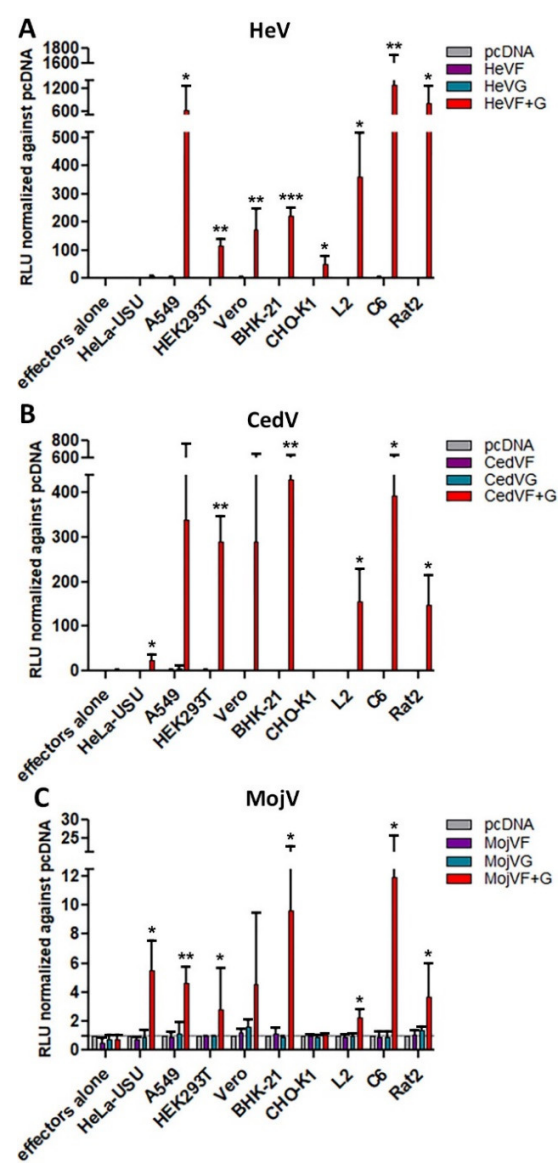

Figure 3. MojV F and G can mediate cell-cell fusion with human, non-human primate and rodent cells in a quantitative Nano luciferase reporter cell-cell fusion assay. BSR-T7/5 effector cells that constitutively express T7 polymerase were transfected with empty vector, henipavirus $\mathrm{F}$ alone, henipavirus $\mathrm{G}$ alone or co-transfected with henipavirus F and G. Human, non-human primate and rodent target cells transfected with the pTM1-Nluc reporter plasmid were incubated with (A) $\mathrm{HeV}$, (B) CedV or (C) MojV effector cells. Effector/target cells mixtures were harvested $24 \mathrm{~h}$, $48 \mathrm{~h}, 72 \mathrm{~h}, 96 \mathrm{~h}, 120 \mathrm{~h}$, day 6 and day 7 post-application of target cells to effector monolayers and assayed for luciferase activity at the time of harvest. Cell-cell fusion was indirectly measured by recording Relative Luminescence Unit (RLU) upon application of Nano-Glo ${ }^{\circledR}$ substrate. Normalized maximum RLUs detected for each cell line are presented here. Maximum RLUs were recorded $48 \mathrm{~h}$ post-application of target cells to effector monolayers co-expressing $\mathrm{HeV}$ (A) or CedV (B) F and G constructs. Effector cells co-transfected with MojV (C) F and G constructs yielded maximum RLUs on day 4 with C6 and Rat2 cells; day 5 with HeLa-USU, A549, HEK293T and L2 cells; and day 6 with Vero and BHK-21 cells. Maximum RLUs normalized against values obtained from target cells co-incubated with effector cells transfected with empty vector are reported here. The grey line represents the normalized reference level of 1 (background). Experiments were repeated at least three times in three technical replicates. The error bars represent the standard deviation. Data were analyzed by Welch's t-test to compare normalized maximum RLUs obtained from each target cell line co-cultured with $\mathrm{F}$ and $\mathrm{G}$ transfected effector cells to that of target cells co-cultured with effector cells expressing F alone. ${ }^{*} p \leq 0.05,{ }^{* *} p \leq 0.01,{ }^{* * *} p \leq 0.001$. 
A549, HEK293T, Vero, BHK-21, as well as L2, C6 and Rat2 cells, were permissive to both $\mathrm{HeV}$ and CedV F and G-mediated cell-cell fusion (Figure 3A,B). MojV F and G mediated varying levels of cell-cell fusion with human (A549, HEK293T, HeLa-USU), non-human primate (Vero) and rodent (BHK-21, L2, C6, Rat2) cells (Figure 3C). The highest levels of cell-cell fusion measured were observed with C6 rat brain cells (Figure 3C). The Nano luciferase reporter gene cell-cell fusion assay revealed that maximum luminescence levels were reached much faster in those cell cultures containing $\mathrm{HeV}$ or CedV F and G expressing effector cells (within $48 \mathrm{~h}$ ) as compared to cultures containing effector cells expressing MojV F and G (within 4 to 6 days) (Figure 3). Furthermore, the levels of MojV F and G-mediated cell-cell fusion were markedly lower than those generated by $\mathrm{HeV}$ or CedV F and G (Figure 3), in agreement with the less extensive syncytial formations observed by microscopy (Figure 2). However, although MojV F and G glycoprotein-mediated cell fusion was less robust and slower to develop, these results demonstrated that additional target cells of human, non-human primate and rodent origin, particularly the rat brain cell line C6, were permissive to MojV F and G glycoprotein-mediated fusion.

\subsection{Role of A- and B-Class Ephrin Ligands in MojV F and G Mediated Membrane Fusion}

MojV was identified through sequencing data obtained from Rattus flavipectus samples [17], and since CedV G can utilize mouse ephrin-A1 ligand as a receptor [31] in addition to human ephrin-A2, -A5, -B1 and -B2, the possibility that MojV G may utilize rodent and/or human ephrin ligands other than ephrin-B2 and -B3, which MojV G does not recognize [39], was examined. Co-precipitation assays were performed to assess protein/protein interaction between a soluble tetrameric form of MojV G (MojV sG) (Figure S4A) and a panel of A- and B-class ephrin ligands, and included soluble tetrameric CedV G (CedV $\mathrm{sG}$ ) [31] and soluble tetrameric GhV G (GhV sG) (Figure S4C) glycoproteins as controls. The results from these co-precipitation assays with CedV sG were congruous with the observed cell fusion mediated by CedV F and G with cells expressing mouse ephrin-A1; human ephrin-A2, -A5, -B1 and -B2 [31]; and CedV G co-precipitated with soluble mouse ephrinA1, -A2, -B1 and -B2; and human ephrin-A5 (Figure S2F) [31]. GhV sG co-precipitated only with ephrin-B2 (Figure S2F,G) in agreement with previous reports $[30,37,38]$. No interactions between MojV sG and any of the ephrin ligands tested were detected by coprecipitation (Figure S2F,G). However, because such low and slow MojV F and G-mediated cell fusion was recorded in the syncytia (Figure 2) and Nano luciferase assays (Figure 3C), the possibility that weak or transient interactions between MojV G and an ephrin ligand capable of triggering $\mathrm{F}$ glycoprotein-mediated fusion, yet not measurable by co-precipitation, could not be excluded. Therefore, a panel of A- and B-class ephrin ligands was tested using the Nano luciferase reporter cell fusion assay with BSR-T7/5 effector cells co-expressing MojV F and $G$ and target cells expressing individual ephrin ligands.

Sequence alignment of the G-H loop of all mouse and human ephrin ligands revealed differences only between mouse and human ephrin-A1 [31] and ephrin-A4. It was previously demonstrated that CedV can utilize mouse ephrin-A1 but not human ephrin-A1, which differs by 1 residue at position 115 within the critical G-H loop region [31]. This single amino acid difference was responsible for supporting CedV F and G-mediated fusion [31]. Additionally, amino acid residues in two ephrin G-H loop positions important for binding to henipavirus $\mathrm{G}$ glycoproteins were noted when comparing mouse to human ephrin-A4 sequences. Here, human ephrin-A4 F115 is replaced by Y118 in mouse ephrin$\mathrm{A} 4$, and both are hydrophobic aromatic residues; this substitution would presumably not significantly impact binding to a henipavirus $\mathrm{G}$ glycoprotein, whereas nucleophilic residue S119 in human ephrin-A4 is replaced by hydrophobic residue P122 in mouse ephrin-A4. Further, the only difference noted between critical residues within the human and rat G-H loop sequences across all eight A- and B-class ephrin ligands was the same P residue. Therefore, mouse ephrin-A1 and rat ephrin-A4 were included in the otherwise all human ephrin ligand panel examined by the Nano luciferase cell-cell fusion assay. 
CHO-K1 cells were chosen as target cells because they are non-permissive to MojV and CedV F and G-mediated cell-cell fusion (Figure 3B,C) [31]. CedV F and G expressing effector cells were used as a control and the resulting cell fusion data were congruent with earlier findings showing permissive fusion with mouse ephrin-A1 and human ephrin-A2, -A5, -B1 and -B2 (Figure 4A) [31]. MojV F and G-mediated cell fusion was detected with target cells expressing rat ephrin-A4, human ephrin-A5 and mouse ephrin-A1, but again at much lower levels and slower kinetics (Figure 4B).

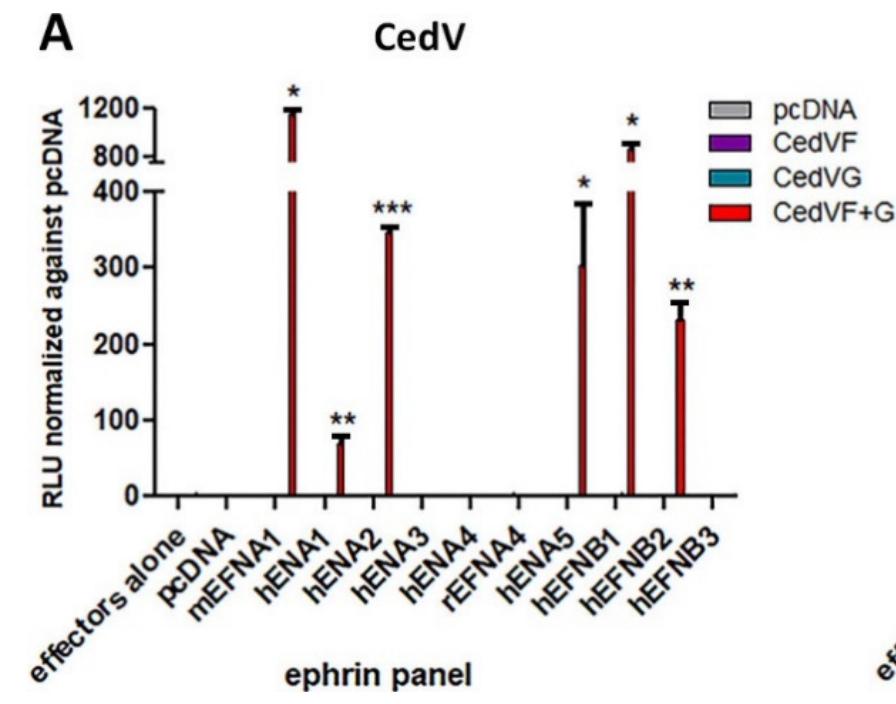

B MojV

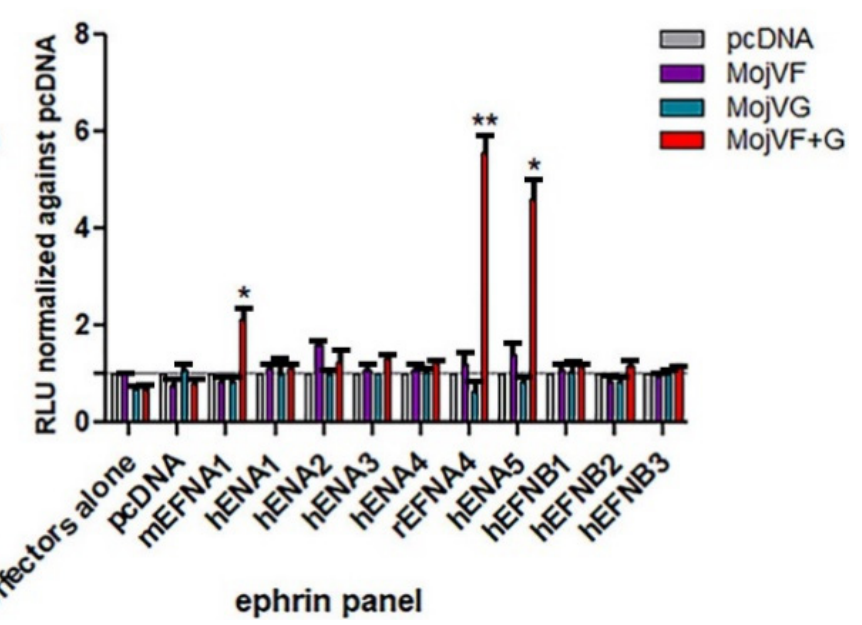

Figure 4. Weak MojV F and G-mediated cell-cell fusion is detected with cells expressing human and rodent A-class ephrin ligands. Cell-cell fusion reporter assays were performed as described in Figure 3 with the following modifications. CHO-K1 target cells were co-transfected with the pTM1-Nluc reporter plasmid and one ephrin-expressing plasmid at a time or an empty vector (pcDNA). Cell mixtures of effector and target cells were assayed for Nano luciferase activity. (A) CedV F and G or (B) MojV F and G effector cells were tested for their ability to mediate cell-cell fusion against a panel of target cells transiently expressing human and rodent ephrin ligands. Maximum RLUs were recorded 48h post-application of target cells to effector monolayers co-expressing CedV F and G constructs. Co-cultures containing effector cells co-transfected with MojV F and G constructs yielded maximum RLUs on day 6. The grey line represents the normalized reference level of 1 (background). The experiments were performed at least 3 times in technical triplicates. m: mouse ephrin ligand; h: human ephrin ligand; r: rat ephrin ligand. Data were analyzed by Welch's $t$ test comparing the normalized maximum RLU means of $\mathrm{F}$ and $\mathrm{G}$ expressing effector cells co-cultured with target cells transfected with an ephrin construct to effector cells co-cultured with target cells transfected with an empty vector (pcDNA). The error bars represent the standard deviation. ${ }^{*} p \leq 0.05,{ }^{* *} p \leq 0.01,{ }^{* * *} p \leq 0.001$.

To further analyze a role of the differing amino acid residue P122/S119 in rat ephrin-A4 versus human ephrin-A4 in supporting this low level of cell fusion, a mutant human ephrinA4 with a G-H loop sequence mimicking the rat ephrin-A4 G-H loop (hEFNA4 S119P) and the converse mutant in rat ephrin-A4 (rEFNA4 P122S) were generated (Figure 5A) and tested in the cell fusion assay. However, mimicking the human G-H loop in the context of the rat ephrin-A4 protein did not abrogate the low and slow level of cell fusion and, introducing the rat G-H loop sequence into the human ephrin-A4 protein did not restore fusion (Figure 5B). There was also no significant difference in MojV F and Gmediated fusion levels with target cells expressing rat ephrin-A4 and rat ephrin-A4 P122S (Figure 5B). In addition, co-precipitation experiments using MojV sG with soluble rat ephrin-A4 (Figure S2D,E) failed to show any binding (Figure S2F,G). Additional Biolayer Interferometry (BLI) assays were performed to assess any possible specific protein/protein interactions; however, no significant binding of MojV sG to rat ephrin-A4, mouse ephrin-A1 or human ephrin-A4, -A5 was detected (Figure S2G). Together, these findings suggest that if A-class ephrin ligands play a role in MojV F and G-mediated cell fusion, they most likely do not act as primary functional receptor(s) for MojV. 
A

\section{Ephrin-A4}

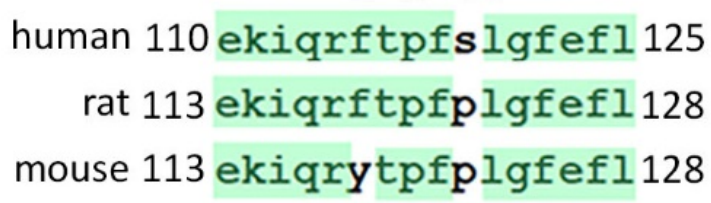

B

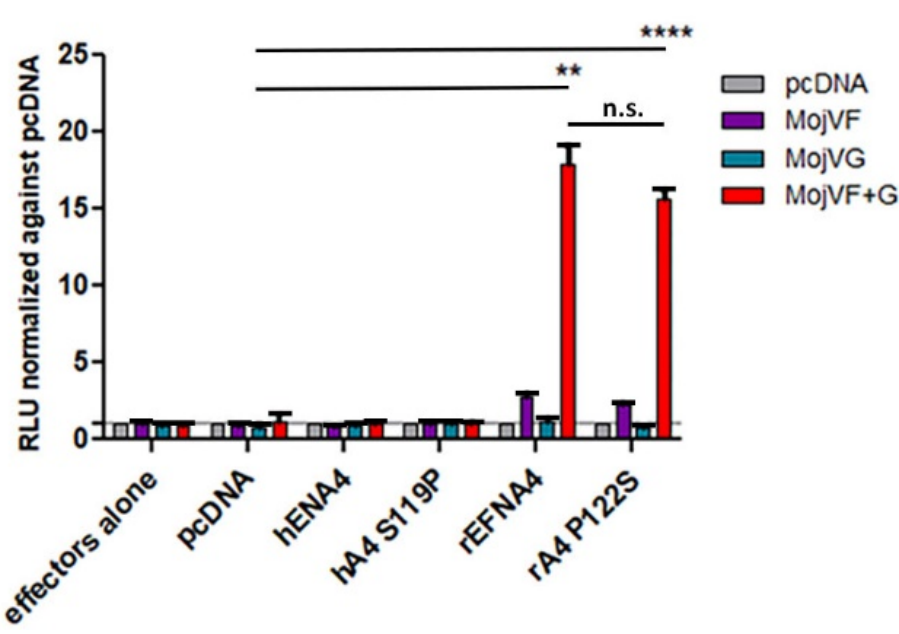

Figure 5. Contribution of rat ephrin-A4 residue P122 to functional interactions with MojV G. (A) Sequence alignment of critical residues $\left.{ }^{*}\right)$ of the G-H loop of human, rat and mouse ephrin-A4 ligand. (B) Cell-cell fusion Nano luciferase assay assessing MojV F and G-mediated fusion with CHO-K1 target cells transiently expressing wild-type or mutant human or rat ephrin ligand A4. m: mouse ephrin ligand; h: human ephrin ligand; r: rat ephrin ligand. Co-cultures containing effector cells co-transfected with MojV F and G constructs yielded maximum RLUs on day 6 . The experiments were performed at least 3 times in technical triplicates. A representative experiment is shown here. Data were analyzed by Welch's $t$ test as described in Figure 4. The error bars represent the standard deviation. ${ }^{* *} p \leq 0.01,{ }^{* * * *} p \leq 0.0001$. n.s. not significant.

\subsection{Oligomerization Status of MojV F and MojV G}

To investigate whether the low cell fusion levels and slow kinetics observed were the result of major structural defects, the oligomeric status of MojV F and G was evaluated in a protein cross-linking assay using the membrane impermeable reagent bis(sulfosuccinimidyl)suberate (BS3). BSR-T7/5 cells transfected with HeV, CedV and MojV F or G constructs were treated with increasing concentrations of BS3; S-tagged proteins were precipitated and analyzed under reducing conditions by SDS-PAGE and Western blotting (Figure 6). Although the efficiency of cross-linking was poor in this assay, MojV F glycoprotein was detected as an oligomer with an apparent trimeric molecular weight of $\sim 180 \mathrm{kDa}$ similar to $\mathrm{CedV} \mathrm{F}$ and $\mathrm{HeV} \mathrm{F}$ (Figure 6A). The HeV, CedV and MojV G glycoproteins were all detected as tetrameric oligomers at the cell surface (Figure 6B). 
A

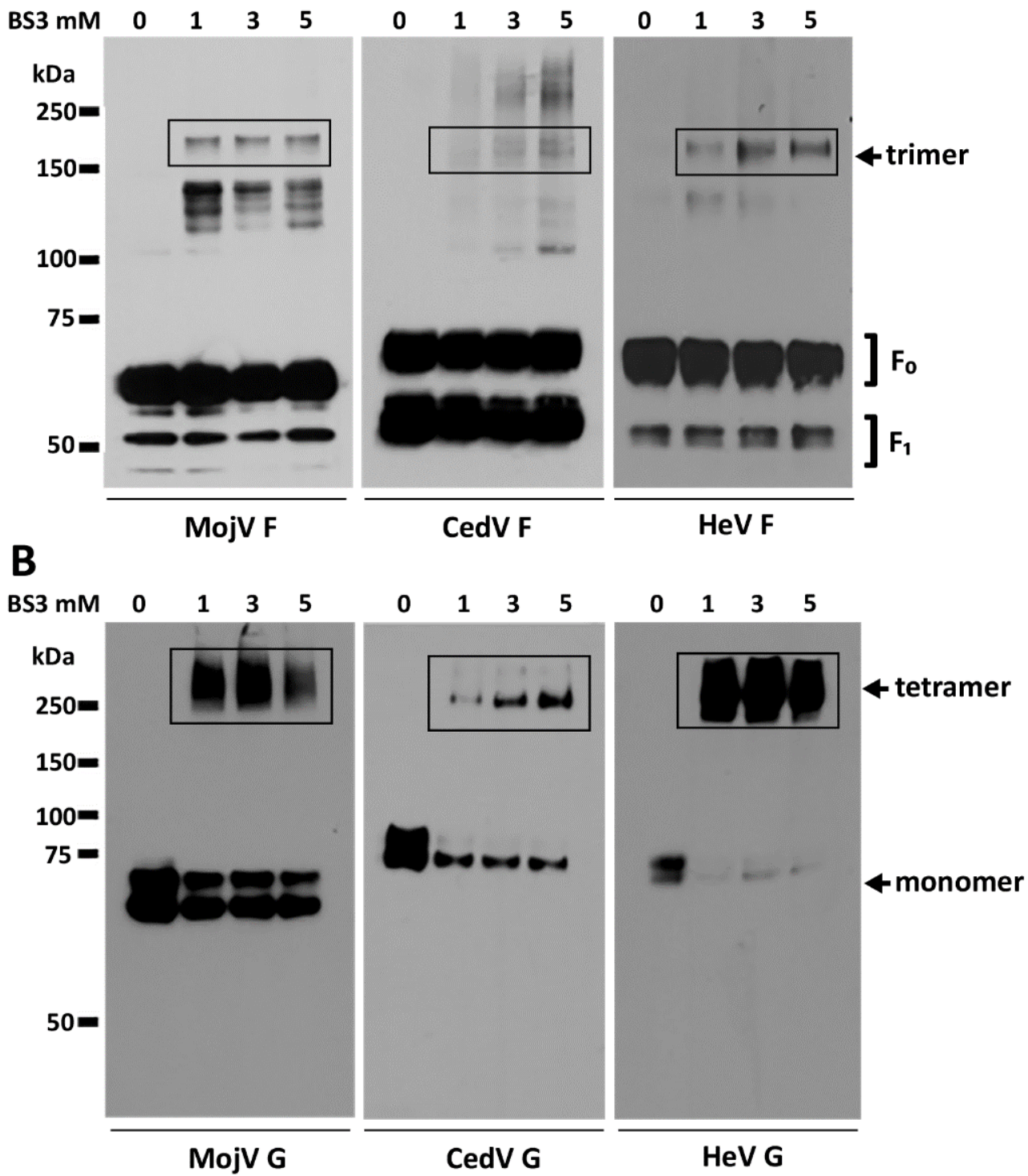

Figure 6. MojV F and G are expressed as trimers and tetramers, respectively, at the cell surface. The oligomerization status of cell-surface-expressed (A) MojV F and (B) MojV G was assessed by treating cells transfected with each construct with increasing concentrations $(0,1,3,5 \mathrm{mM})$ of the cell-surface protein cross-linking reagent BS3. Cross-linked S-tagged proteins were precipitated with S agarose beads. The precipitates were resolved by SDS-PAGE and analyzed by Western blot under reducing conditions. $\mathrm{HeV}$ and $\mathrm{CedV}$ recombinant glycoproteins were included as controls.

\subsection{MojV Soluble F Glycoprotein Pre- to Post-Fusion Conformational Change}

The possibility that a defect in the ability of the MojV F glycoprotein to undergo a preto post-fusion conformational change required for efficient cell fusion was investigated using a previously developed HR2 heptad peptide triggering and capture assay [45,48]. The F glycoproteins of henipaviruses, upon triggering, adopt an intermediate conformation that establishes a protein bridge between the virion and host cell membranes (reviewed in [24,25]). In this state, the heptad repeat HR1 and HR2 domains of each $\mathrm{F}_{1}$ subunit within the F trimer can interact and fold, leading to the formation of a six-helix bundle hairpin $(6 \mathrm{HB})$ structure that promotes the subsequent merging of the virion and cellular membranes and virus infection. Previous studies established that recombinant pre-fusion conformation forms of trimeric soluble $\mathrm{HeV} \mathrm{F}(\mathrm{HeV} \mathrm{sF})$ and $\mathrm{NiV} \mathrm{F}(\mathrm{NiV} \mathrm{sF})$ glycoproteins could be triggered in vitro to refold into post-fusion conformations by application of trypsin 
and heat [45]. During the conformational transition of $\mathrm{sF}$ from its pre- to post-fusion form, if biotinylated HR2 peptides (FC2 peptides) homologous to $\mathrm{HeV}$ and NiV F were present, the labeled peptide would bind and capture the $\mathrm{sF}$ trimer in an intermediate state between pre- and post-fusion conformations [45]. To establish that MojV F was similarly capable of measurable activation, triggering and transition from a pre- to post-fusion conformation, a soluble construct of MojV F was prepared (MojV sF) along with CedV F (CedV sF) (Figure S1A) as an additional control as previously performed with $\mathrm{HeV}$ and $\mathrm{NiV}$ sF constructs $[44,45,48]$. The expression and oligomerization status of the MojV and CedV sF constructs were confirmed by Western blot analysis (Figure S1B) and protein cross-linking assays (Figure S1C). Using purified MojV and CedV sF trimers, the HR2 heptad peptide triggering and capture assay was conducted. Similar to $\mathrm{HeV}$ and $\mathrm{NiV} \mathrm{sF}$ constructs, the $\mathrm{Moj} \mathrm{V}$ and $\mathrm{CedV} \mathrm{sF}$ are expressed as predominantly unprocessed trimeric $\mathrm{F}_{0}$ precursors (Figure S1B and C), but batch to batch sF preparations can exhibit some cleavage into the $\mathrm{F}_{1} / \mathrm{F}_{2}$ subunits. To mimic the proteolytic processing of the $\mathrm{F}_{0}$ precursor into its $\mathrm{F}_{1}$ and $\mathrm{F}_{2}$ subunits, MojV and CedV sF were digested with trypsin and heat was used to trigger their pre- to post-fusion conformational change. The $\mathrm{sF}$ trimers were treated with various orders of application of trypsin and heat in presence and absence of their respective FC2 peptides, followed by avidin agarose bead precipitation and SDS-PAGE and Western blot analysis (Figure 7).

Similar to the findings reported for $\mathrm{HeV}$ and $\mathrm{NiV}$ sF [45], a combination of trypsin followed by addition of FC2 peptide then followed by heat application can capture a conformational intermediate of $\mathrm{MojV}$ and $\mathrm{CedV} \mathrm{sF}$ as the trimer transitions from a prefusion to a post-fusion state. As was shown for $\mathrm{NiV}$ and $\mathrm{HeV} \mathrm{sF}$ [45], it was noted that only trypsin-processed CedV sF could be triggered into this conformational transition by heat treatment and captured by the FC2 peptide (Figure 7B). Interestingly, however, MojV sF could also be captured after heat application in the presence of FC2 peptide without trypsin treatment (Figure 7B). Although this preparation of MojV sF trimer was apparently partially processed into the $F_{1}$ and $F_{2}$ subunits, it suggested that the pre-fusion conformation of the MojV sF might be less stable in comparison to other henipavirus $\mathrm{sF}$ trimers. An analysis of purified preparations of MojV and CedV sF oligomers by nsEM revealed that the glycoproteins were monodispersed trimers, but were primarily in postfusion conformations when immobilized onto the grids (Figure S4B,D). Both pre-fusion and post-fusion conformations of trimeric MojV sF were identified (Figure S4B) and only post-fusion conformation of CedV sF was observed with a new preparation (Figure S4D). Although this could suggest some conformation instability of these sF species designed and prepared here, variations in the amounts of pre-fusion and post-fusion conformations of individual sF preparations do occur. We have also observed $\mathrm{sF}$ trimers of $\mathrm{NiV}$ and $\mathrm{HeV}$ fold to a post-fusion conformation upon freez-thaw or when immobilized onto ELISA plates, as indicated from a loss of recognition by a pre-fusion specific mAb known as 5B3 [45,48]. We believe the heptad peptide triggering and capture assay using sF glycoproteins in solution is a reliable assessment of the pre- to post-fusion conformational change of a sF glycoprotein preparation. 
A
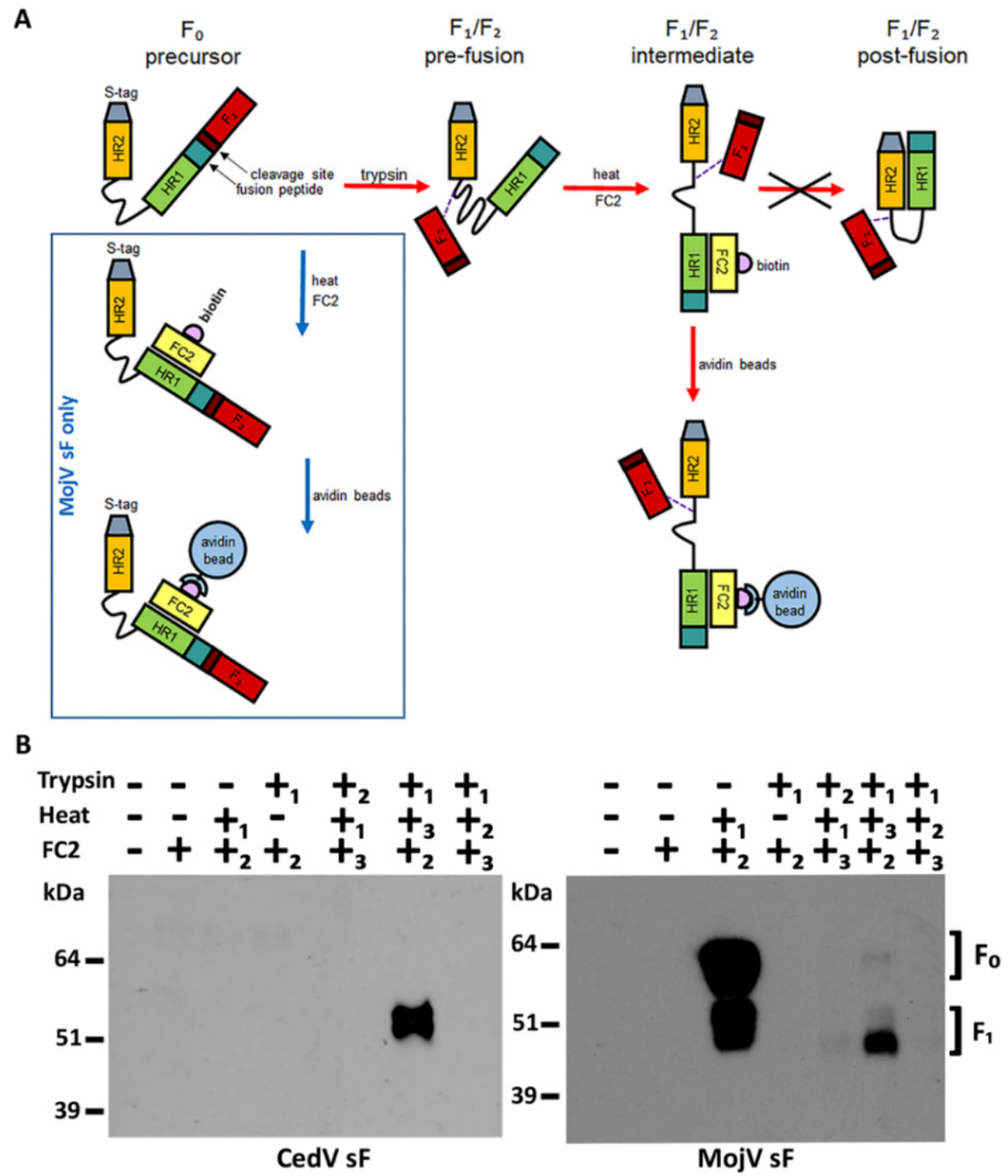

Figure 7. MojV sF can be triggered to undergo a pre- to post-fusion conformational change. (A) HR2 heptad peptide triggering and capture assay. The henipavirus $\mathrm{sF}_{0}$ is cleaved into the disulfide linked $\mathrm{sF}_{1}$ and $\mathrm{F}_{2}$ subunits by trypsin digestion. The pre-fusion conformation of cleaved $\mathrm{sF}$ trimer is triggered into transitioning from pre- to post-fusion conformation by heat application. Upon triggering, prefusion $\mathrm{sF}$ rearranges into an intermediate structure before reaching its postfusion conformation. This intermediate conformation allows access to the HR1 domain of $\mathrm{sF}_{1}$ by a biotinylated HR2 peptide (FC2). Interaction between intermediate stage $\mathrm{sF}_{1}$ and the FC2 peptide prevents $\mathrm{sF}$ from completing its transition into the post-fusion conformation by blocking HR1/HR2 interactions. The FC2 peptide thus "captures" an intermediate conformation of sF as it transitions from pre- to postfusion conformation. The sF/FC2 peptide complex can then be precipitated by incubation with avidin beads. The dashed line represents the disulfide bond between the $F_{1}$ and $F_{2}$ subunits. (B) Soluble MojV F (MojV sF) and CedV F (CedV sF) proteins were treated with various combinations of trypsin, heat and the addition of FC2 peptide. The order of treatment applied in each combination in numbered 1,2,3. Treated protein complexes were precipitated with avidin agarose beads, resolved under reducing conditions by SDS-PAGE and analyzed by Western blot. CedV sF was detected with mouse polyclonal anti-CedV sF serum at a 1:1000 dilution. MojV sF was detected with mAb 4G5 at a 1:2500 dilution. The $F_{2}$ is not detected. This experiment was performed more than 5 times with different preparations of $\mathrm{sF}$ glycoprotein trimers. 


\subsection{The Low and Slow MojV F Mediated Fusion Phenotype Is Not Attributable to a Defect in Function}

Co-expressed heterotypic combinations of $\mathrm{HeV}, \mathrm{NiV}$ and $\mathrm{GhV} F$ and $\mathrm{G}$ have been shown to retain functionality and mediate cell fusion [60,62]. Here, the respective contribution of the MojV F and G glycoproteins to the cell fusion process was examined by heterotypic F/G glycoprotein cell fusion assays to further evaluate the MojV F glycoprotein fusion phenotype. Individual BSR-T7/5 effector cell populations were produced using each henipavirus $\mathrm{F}$ glycoprotein paired individually with each henipavirus $\mathrm{G}$ glycoprotein, and C6 rat glial cells were used as target cells since this cell line supported the highest levels of $\mathrm{MojV}$-mediated cell fusion and was also permissive for CedV and $\mathrm{HeV}-$ mediated cell fusion. The Nano luciferase reporter gene cell-cell fusion assay was conducted simultaneously with all cell combinations (Figure 8).

A

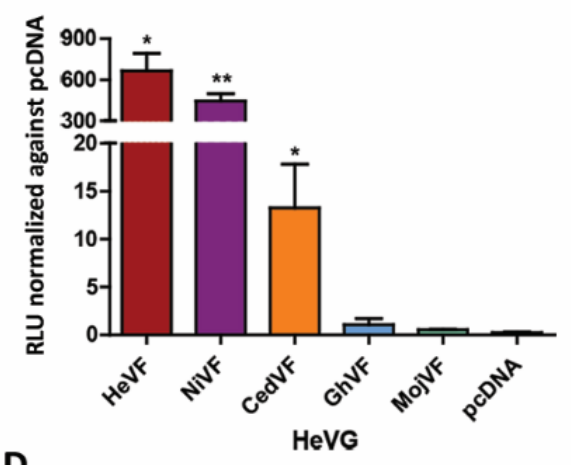

D

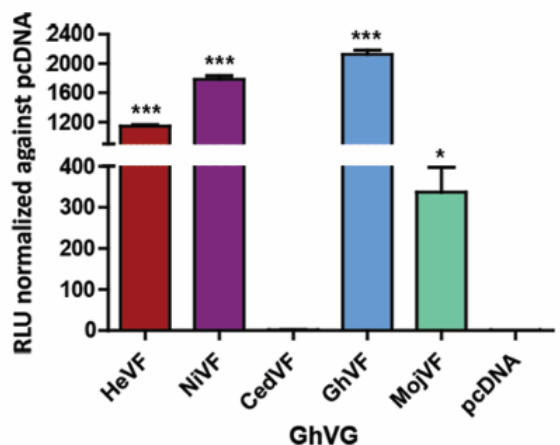

B

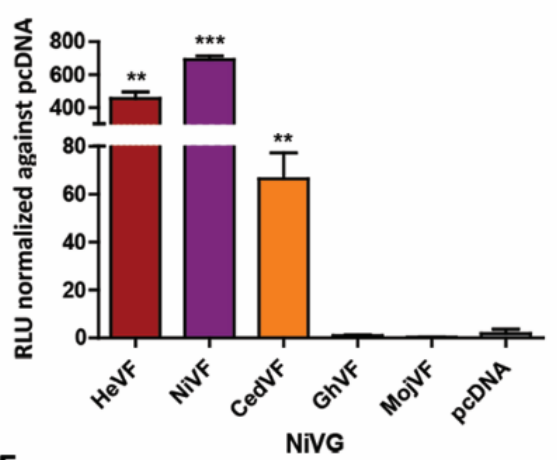

E

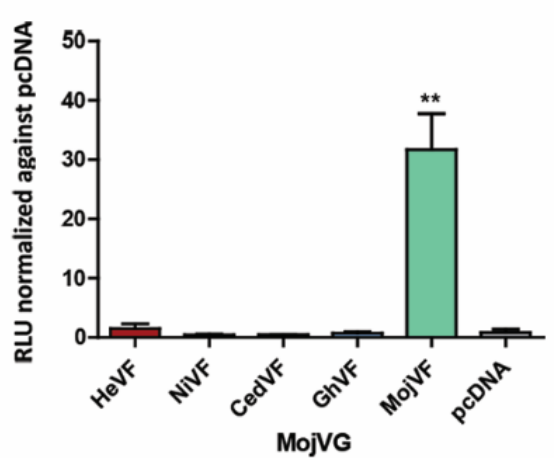

C

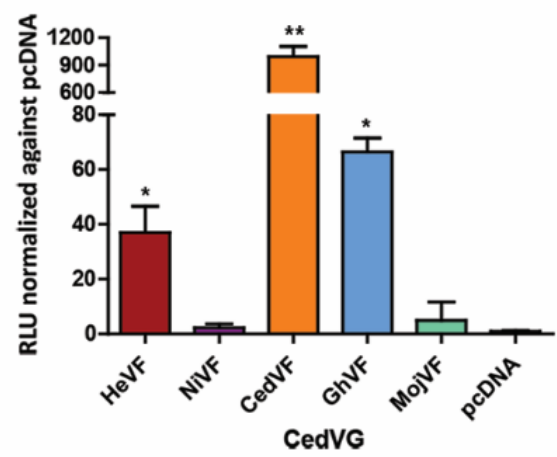

Figure 8. Heterotypic henipavirus F and G complementation assay confirms MojV F functionality in a quantitative Nano luciferase reporter cell-cell fusion assay. BSR-T7/5 effector cells were co-transfected with empty vector or henipavirus $\mathrm{F}$ and either (A) HeV G, (B) NiV G, (C) CedV G, (D) GhV G or (E) MojV G. Effector cells were co-incubated with C6 rat glial cells transfected with the pTM1-Nluc reporter plasmid. Maximum RLUs were recorded $48 \mathrm{~h}$ post-application of target cells to all effector monolayers, except for co-cultures with effector cells expressing MojV G, which reached maximum RLUs on day 4. Maximum RLUs were normalized against background defined as RLUs from mixtures of the target C6 cells applied to effector cells co-transfected with henipavirus $\mathrm{G}$ and empty vectors. The experiments were performed at least 3 times in technical triplicates. A representative experiment is shown here. Data were analyzed by Welch's $t$ test. The error bars represent the standard deviation. ${ }^{*} p \leq 0.05,{ }^{* *} p \leq 0.01,{ }^{* * *} p \leq 0.001$.

Heterotypic combinations of $\mathrm{HeV}$ and $\mathrm{NiV} \mathrm{F}$ and $\mathrm{G}$ were functionally competent in a bidirectional manner as previously reported [60]. In addition, $\mathrm{HeV} \mathrm{G}$ and NiV G successfully triggered CedV F to mediate cell-cell fusion, albeit at lower levels than their homotypic pairings and the heterotypic $\mathrm{HeV} / \mathrm{NiV}$ pairings (Figure $8 \mathrm{~A}-\mathrm{C}$ ). Co-expression of NiV F with CedV G did not result in cell fusion, but the HeV F/CedV G paring was cell fusion competent. The CedV G/MojV F and the CedV F/MojV G pairings were not cell fusion competent. (Figure 8E). The MojV F and G-mediated cell fusion was again functional, but notably the MojV F and GhV G pairing was also cell fusion competent as previously 
reported (Figure 8D) [39]. However, the level of MojV F and GhV G-mediated cell fusion was increased 10-fold and did not display the slow cell fusion phenotype exhibited by the homotypic MojV F and $G$ pairing-mediated cell fusion. Whereas the maximum fusion levels mediated by the homologous MojV F and G pairing were recorded on day 4, those mediated by the heterologous pairing MojV F/GhV G were observed at the $48 \mathrm{~h}$ time point. Together, with the earlier biochemical and functional analyses, these data indicate that the low and slow fusion phenotype of the MojV F and G glycoproteins is not due to a functional defect in the MojV F but more likely due to MojV G receptor restrictions.

\section{Discussion}

In the absence of an infectious isolate of MojV, which is known only from sequence data, details about the cellular and species tropism, receptor use and infection process, as well as any pathogenic potential of this henipavirus, remain quite limited. Over the past more than two decades, studies using henipavirus $F$ and $G$ glycoproteins, produced by recombinant techniques, as surrogate for infectious virus, have provided a wealth of information on the infection tropism and virus-host cell interactions of henipaviruses. Here, we investigated the functional and biochemical characteristics of the $F$ and $G$ glycoproteins of MojV. Using established techniques, we demonstrated that, although functional, MojV F and G-mediated cell fusion possessed a low and slow cell fusion phenotype when compared to $\mathrm{HeV}$ and $\mathrm{CedV}$, which was in agreement with observations previously reported on MojV F and G-mediated cell fusion that was described as "significantly less robust" than cell fusion mediated by NiV-F and G [39]. In addition to confirming previous findings with human HEK293T and A549 and rodent BSR-T7/5 and BHK-21 cells [39], we found that MojV F and G-mediated cell fusion was permissive with several other cell lines of human (HeLa-USU), non-human primate (Vero) and rodent origin (L2, Rat2 and C6). An exhaustive analysis of potential ephrin ligand interaction with MojV G and comparison to that of CedV G, which was earlier shown capable of a functional interaction with mouse ephrin-A1 [31], found no significant binding interactions between MojV G and any A-class or B-class ephrin proteins tested in co-precipitation and BLI assays. Although we detected very low levels of apparently specific cell fusion mediated by MojV F and G glycoproteins with cells expressing mouse ephrin-A1, rat ephrin-A4 and human ephrin-A5, the low levels and slow kinetics of cell fusion do not support a conclusion that these proteins are natural entry receptors for MojV.

Potential biochemical or structural defects in the recombinant expressed MojV F and G glycoproteins that could account for the low and slow cell fusion phenotype observed here and in previous studies [39] were not identified. The data here showed that recombinant MojV F and $G$ share similarities in their expression and migration patterns in comparison to $\mathrm{HeV}$ and $\mathrm{CedV} F$ and $\mathrm{G}$, suggesting proper structure and folding, and recapitulated the higher order oligomerization status of $F$ and $G$ seen with other henipavirus $F$ and $G$ glycoproteins. In addition, an HR2 heptad peptide triggering and capture assay provided evidence that recombinant $\mathrm{MojV} s \mathrm{~s}$ is functionally competent with a similar pre- to postfusion conformational transition capacity as previously observed with $\mathrm{NiV}$ and $\mathrm{HeV}$ sF [45,48] and with CedV sF shown here. Notably, however, MojV sF trimers, in apparently both pre- and post-fusion conformations, of predominantly unprocessed $\mathrm{F}_{0}$ subunits could be readily detected in the HR2 heptad peptide triggering and capture assay just by raising the temperature. Indeed, this observation did lead to an initial speculation that the observed low and slow cell fusion phenotype may be the result of having a significant proportion of the recombinantexpressed MojV F on the cell surface already in a post-fusion or partially triggered form. However, an analysis of the respective contribution of the MojV F and $\mathrm{G}$ glycoproteins to the cell fusion process revealed that MojV F facilitated significant cell fusion in the context of its triggering by GhV G, known to engage with ephrin-B2 as its functional receptor $[30,38]$, in comparison to its homologous MojV G glycoprotein. Additionally, the possibility that the instability observed here with recombinant MojV sF is artificial cannot be excluded because the $\mathrm{sF}$ trimer is removed from the context of an 
intact virion. Indeed, work by Cifuentes-Muñoz et al. suggests that the $\mathrm{HeV} F$ and the virus matrix $(\mathrm{M})$ proteins pre-assemble before they reach the plasma membrane [63]. Taken together, the findings presented here suggest that the low and slow cell fusion phenotype exhibited by the MojV F and G glycoproteins likely result from entry receptor restrictions rather than an inherent functional defect in MojV F. The possibility that recombinant MojV $G$ encodes defects that prevent proper receptor engagement also cannot be ruled out, and such defects may be present owing to the discovery of MojV by genetic sequence data alone. The natural fusion-triggering and entry receptor for MojV may be expressed at levels too low, on the various cell lines tested in the present study, for robust cell fusion to be detected. Experiments here focused on examining the susceptibility of cells of primate and rodent origin to MojV F and G-mediated membrane fusion and while Pteropus bats are not found in China, non-Pteropus species such as bats of the genus Rousettus might be potential reservoirs for MojV. An examination of MojV F and G-mediated syncytia formation and fusion kinetics in non-Pteropus bat cells will be important. However, the results here do suggest that neither A- nor B-class ephrin ligands serve as primary functional receptors of MojV.

Nevertheless, the discovery and characterization of both CedV and MojV suggest the possibility of additional natural hosts for the genus Henipavirus. Indeed, although mice were initially reported as resistant to $\mathrm{HeV}$ and $\mathrm{NiV}$ infections [64,65], Dups et al. [66] established a model of $\mathrm{HeV}$ encephalitis in aged mice infected by intranasal inoculation. Further, the golden hamster is now a well-accepted model of $\mathrm{NiV}$ and $\mathrm{HeV}$ infection and pathogenesis [65,67]. Additionally, the A-class rodent ephrin ligand (mouse ephrin-A1) supports cell fusion and infection by CedV [31]. In addition, the present study shows that rat cells are susceptible to $\mathrm{HeV}, \mathrm{CedV}$ and MojV F and G-mediated cell fusion, with rat C6 glial cells serving as a particularly competent fusion permissive cell line with MojV G and F glycoproteins. That rodents could serve as natural hosts or virus amplifiers of CedV and MojV therefore appears possible.

The type I INF response antagonists $\mathrm{V}$ and $\mathrm{W}$ proteins have been shown to play a critical role in $\mathrm{NiV}$ and $\mathrm{HeV}$ pathogenesis [68,69]. Analysis of the MojV genomic data reveals the RNA editing sites that would allow for the expression of MojV V and W proteins [17], which the non-pathogenic CedV lacks [69]. The pathogenic potential of MojV remains unknown at present, but could be explored by virus rescue using reverse genetics techniques under BSL-4 containment, and the present studies have provided some insight into the functional characteristics of the critically important MojV structural glycoproteins that could facilitate virus rescue studies.

Supplementary Materials: The following are available online at https://www.mdpi.com/1999 -4915/13/3/517/s1, Figure S1: Design, expression and oligomerization of MojV sF; Figure S2: Design, expression and oligomerization status of MojV and GhV sG glycoproteins, soluble rat ephrin-A4-Fc expression and protein/protein interaction assessment by co-precipitation and biolayer interferometry assays; Figure S3: Size exclusion chromatography, blue native (BN) PAGE and Western blot analyses of soluble henipavirus G and F glycoproteins; Figure S4: Electron microscopy analysis of MojV and CedV sF, and GhV and MojV sG glycoproteins; Figure S5: Syncytia formation is not detected in MojV F and G transfected Vero and HEK293T cells by microscopy.

Author Contributions: S.C.D.S. and C.C.B. conceived and designed the experiments; S.C.D.S. performed the experiments, analyzed data and drafted the manuscript. S.C.D.S. and L.Y. performed the mouse immunizations. L.Y. prepared the $\mathrm{CedV} \mathrm{sF}$ construct, conducted large scale protein production and generated the monoclonal antibody 4G5. H.V.D. conducted the biolayer interferometry experiments and data analysis; K.X. conducted the negative-staining electron microscopy experiments; C.C.B. provided overall supervision and financial support of the project and revised the manuscript. D.V. and J.H.E. edited the manuscript and provided financial support. C.C.B. revised and prepared the final version of the manuscript. All authors have read and agreed to the published version of the manuscript.

Funding: Research support was provided by Defense Threat Reduction Agency HDTRA1-17-10037 to J.H.E, National Institutes of Health grants AI054715 and AI077995 to C.C.B., the Uniformed Services 
University grant MIC-73-9450 to S.C.D.S.; and National Institutes of Health grants DP1AI158186 and HHSN272201700059C, the National Institute of General Medical Sciences R01GM120553, a Pew Biomedical Scholars Award and Investigators in the Pathogenesis of Infectious Disease Award from the Burroughs Wellcome Fund to D.V.

Institutional Review Board Statement: Mice studies were conducted in accordance with protocol MIC 16-262 approved by the Uniformed Service University Animal Care and Use Committee, 14 September 2016.

Informed Consent Statement: Not applicable.

Data Availability Statement: Not applicable.

Acknowledgments: We thank Eric Laing for his critical review and helpful comments and Stephanie Petzing for her preliminary work on the GhV sG construct. The views expressed in the manuscript are solely those of the authors and do not represent official views or opinions of the Department of Defense or the Uniformed Services University of the Health Sciences.

Conflicts of Interest: The authors declare no conflict of interest. The funders had no role in the design of the study; in the collection, analyses, or interpretation of data; in the writing of the manuscript, or in the decision to publish the results.

\section{References}

1. Wang, L.; Mackenzie, J.S.; Broder, C.C. Henipaviruses. In Fields Virology; Knipe, D., Ed.; Wolters Kluwer Health/Lippincott Williams \& Wilkins: Philadelphia, PA, USA, 2013; pp. 1070-1085.

2. Chua, K.B.; Bellini, W.J.; Rota, P.A.; Harcourt, B.H.; Tamin, A.; Lam, S.K.; Ksiazek, T.G.; Rollin, P.E.; Zaki, S.R.; Shieh, W.; et al. Nipah Virus: A recently emergent deadly paramyxovirus. Science 2000, 288, 1432-1435. [CrossRef] [PubMed]

3. De Wit, E.; Munster, V.J. Animal models of disease shed light on Nipah virus pathogenesis and transmission. J. Pathol. 2015, 235, 196-205. [CrossRef]

4. Amaya, M.; Broder, C.C. Vaccines to emerging viruses: Nipah and Hendra. Annu. Rev. Virol. 2020, 7, 447-473. [CrossRef] [PubMed]

5. Geisbert, T.W.; Feldmann, H.; Broder, C.C. Animal challenge models of Henipavirus infection and pathogenesis. Curr. Top. Microbiol. Immunol. 2012, 359, 153-177. [CrossRef] [PubMed]

6. Eaton, B.T.; Broder, C.C.; Middleton, D.; Wang, L.-F. Hendra and Nipah viruses: Different and dangerous. Nat. Rev. Genet. 2006, 4, 23-35. [CrossRef]

7. Sharma, V.; Kaushik, S.; Kumar, R.; Yadav, J.P.; Kaushik, S. Emerging trends of Nipah virus: A review. Rev. Med. Virol. 2019, 29, e2010. [CrossRef]

8. Luby, S.P.; Hossain, M.J.; Gurley, E.S.; Ahmed, B.-N.; Banu, S.; Khan, S.U.; Homaira, N.; Rota, P.A.; Rollin, P.E.; Comer, J.A.; et al. Recurrent zoonotic transmission of Nipah virus into humans, Bangladesh, 2001-2007. Emerg. Infect. Dis. 2009, 15, 1229-1235. [CrossRef]

9. Luby, S.P.; Gurley, E.S. Epidemiology of Henipavirus disease in humans. Curr. Top. Microbiol. Immunol. 2012, 359, 25-40. [CrossRef]

10. Ching, P.K.G.; de los Reyes, V.C.; Sucaldito, M.N.; Tayag, E.; Columna-Vingno, A.B.; Malbas, F.F.; Bolo, G.C.; Sejvar, J.J.; Eagles, D.; Playford, G.; et al. Outbreak of Henipavirus infection, Philippines, 2014. Emerg. Infect. Dis. 2015, 21, 328-331. [CrossRef]

11. Arunkumar, G.; Chandni, R.; Mourya, D.T.; Singh, S.K.; Sadanandan, R.; Sudan, P.; Bhargava, B.; Gangakhedkar, R.R.; Gupta, N.; Yadav, P.D.; et al. Outbreak investigation of Nipah Virus Disease in Kerala, India, 2018. J. Infect. Dis. 2019, 219, 1867-1878. [CrossRef]

12. Broder, C.C.; Weir, D.L.; Reid, P.A. Hendra virus and Nipah virus animal vaccines. Vaccine 2016, 34, 3525-3534. [CrossRef]

13. Middleton, D.; Pallister, J.; Klein, R.; Feng, Y.-R.; Haining, J.; Arkinstall, R.; Frazer, L.; Huang, J.-A.; Edwards, N.; Wareing, M.; et al. Hendra Virus vaccine, a one health approach to protecting horse, human, and environmental health. Emerg. Infect. Dis. 2014, 20, 372-379. [CrossRef]

14. Broder, C.C.; Xu, K.; Nikolov, D.B.; Zhu, Z.; Dimitrov, D.S.; Middleton, D.; Pallister, J.; Geisbert, T.W.; Bossart, K.N.; Wang, L.-F. A treatment for and vaccine against the deadly Hendra and Nipah viruses. Antivir. Res. 2013, 100, 8-13. [CrossRef] [PubMed]

15. Drexler, J.F.; Corman, V.M.; Gloza-Rausch, F.; Seebens, A.; Annan, A.; Ipsen, A.; Kruppa, T.; Müller, M.A.; Kalko, E.K.V.; Adu-Sarkodie, Y.; et al. Henipavirus RNA in African bats. PLoS ONE 2009, 4, e6367. [CrossRef] [PubMed]

16. Marsh, G.A.; De Jong, C.; Barr, J.A.; Tachedjian, M.; Smith, C.; Middleton, D.; Yu, M.; Todd, S.; Foord, A.J.; Haring, V.; et al. Cedar virus: A novel Henipavirus isolated from Australian bats. PLoS Pathog. 2012, 8, e1002836. [CrossRef]

17. Wu, Z.; Yang, L.; Yang, F.; Ren, X.; Jiang, J.; Dong, J.; Sun, L.; Zhu, Y.; Zhou, H.; Jin, Q. Novel Henipa-like Virus, Mojiang Paramyxovirus, in rats, China, 2012. Emerg. Infect. Dis. 2014, 20, 1064-1066. [CrossRef] [PubMed]

18. Rahalkar, M.C.; Bahulikar, R.A. Lethal pneumonia cases in Mojiang miners (2012) and the mineshaft could provide important clues to the origin of SARS-CoV-2. Front. Public Health 2020, 8, 581569. [CrossRef] [PubMed] 
19. Drexler, J.F.; Corman, V.M.; Müller, M.A.; Maganga, G.D.; Vallo, P.; Binger, T.; Gloza-Rausch, F.; Cottontail, V.M.; Rasche, A.; Yordanov, S.; et al. Bats host major mammalian paramyxoviruses. Nat. Commun. 2012, 3, 796. [CrossRef] [PubMed]

20. Chang, A.; Dutch, R.E. Paramyxovirus fusion and entry: Multiple paths to a common end. Viruses 2012, 4, 613-636. [CrossRef]

21. Bose, S.; Jardetzky, T.S.; Lamb, R.A. Timing is everything: Fine-tuned molecular machines orchestrate paramyxovirus entry. Virology 2015, 479-480, 518-531. [CrossRef]

22. Navaratnarajah, C.K.; Generous, A.R.; Yousaf, I.; Cattaneo, R. Receptor-mediated cell entry of paramyxoviruses: Mechanisms, and consequences for tropism and pathogenesis. J. Biol. Chem. 2020, 295, 2771-2786. [CrossRef]

23. Dutch, R.E. Entry and fusion of emerging paramyxoviruses. PLoS Pathog. 2010, 6, e1000881. [CrossRef] [PubMed]

24. Lee, B.; Ataman, Z.A. Modes of paramyxovirus fusion: A Henipavirus perspective. Trends Microbiol. 2011, 19, 389-399. [CrossRef] [PubMed]

25. Aguilar, H.C.; Iorio, R.M. Henipavirus membrane fusion and viral entry. Curr. Top. Microbiol. Immunol. 2012, 359, 79-94. [CrossRef] [PubMed]

26. Aguilar, H.C.; Henderson, B.A.; Zamora, J.L.; Johnston, G.P. Paramyxovirus Glycoproteins and the membrane fusion process. Curr. Clin. Microbiol. Rep. 2016, 3, 142-154. [CrossRef]

27. Pasquale, E.B. Eph-Ephrin bidirectional signaling in physiology and disease. Cell 2008, 133, 38-52. [CrossRef]

28. Lisabeth, E.M.; Falivelli, G.; Pasquale, E.B. Eph receptor signaling and Ephrins. Cold Spring Harb. Perspect. Biol. 2013, 5, a009159. [CrossRef]

29. Xu, K.; Broder, C.C.; Nikolov, D.B. Ephrin-B2 and ephrin-B3 as functional henipavirus receptors. Semin. Cell Dev. Biol. 2012, 23, 116-123. [CrossRef]

30. Lee, B.; Pernet, O.; Ahmed, A.A.; Zeltina, A.; Beaty, S.M.; Bowden, T.A. Molecular recognition of human ephrinB2 cell surface receptor by an emergent African henipavirus. Proc. Natl. Acad. Sci. USA 2015, 112, E2156-E2165. [CrossRef]

31. Laing, E.D.; Navaratnarajah, C.K.; Cheliout Da Silva, S.; Petzing, S.R.; Xu, Y.; Sterling, S.L.; Marsh, G.A.; Wang, L.-F.; Amaya, M.; Nikolov, D.B.; et al. Structural and functional analyses reveal promiscuous and species specific use of ephrin receptors by Cedar virus. Proc. Natl. Acad. Sci. USA 2019, 116, 20707-20715. [CrossRef]

32. Toth, J.; Cutforth, T.; Gelinas, A.D.; Bethoney, K.A.; Bard, J.; Harrison, C.J. Crystal structure of an Ephrin ectodomain. Dev. Cell 2001, 1, 83-92. [CrossRef]

33. Bonaparte, M.I.; Dimitrov, D.S.; Bossart, K.N.; Crameri, G.; Mungall, B.A.; Bishop, K.A.; Choudhry, V.; Dimitrov, D.S.; Wang, L.-F.; Eaton, B.T.; et al. Ephrin-B2 ligand is a functional receptor for Hendra virus and Nipah virus. Proc. Natl. Acad. Sci. USA 2005, 102, 10652-10657. [CrossRef]

34. Negrete, O.A.; Levroney, E.L.; Aguilar, H.C.; Bertolotti-Ciarlet, A.; Nazarian, R.; Tajyar, S.; Lee, B. EphrinB2 is the entry receptor for Nipah virus, an emergent deadly paramyxovirus. Nature 2005, 436, 401-405. [CrossRef]

35. Negrete, O.A.; Wolf, M.C.; Aguilar, H.C.; Enterlein, S.; Wang, W.; Mühlberger, E.; Su, S.V.; Bertolotti-Ciarlet, A.; Flick, R.; Lee, B. Two key residues in EphrinB3 are critical for its use as an alternative receptor for Nipah virus. PLoS Pathog. 2006, 2, e7. [CrossRef] [PubMed]

36. $\mathrm{Xu}, \mathrm{K} . ;$ Rajashankar, K.R.; Chan, Y.-P.; Himanen, J.P.; Broder, C.C.; Nikolov, D.B. Host cell recognition by the henipaviruses: Crystal structures of the Nipah G attachment glycoprotein and its complex with ephrin-B3. Proc. Natl. Acad. Sci. USA 2008, 105, 9953-9958. [CrossRef] [PubMed]

37. Krüger, N.; Hoffmann, M.; Weis, M.; Drexler, J.F.; Müller, M.A.; Winter, C.; Corman, V.M.; Gützkow, T.; Drosten, C.; Maisner, A.; et al. Surface glycoproteins of an African Henipavirus induce syncytium formation in a cell line derived from an African fruit bat, Hypsignathus monstrosus. J. Virol. 2013, 87, 13889-13891. [CrossRef]

38. Weis, M.; Behner, L.; Hoffmann, M.; Krüger, N.; Herrler, G.; Drosten, C.; Drexler, J.F.; Dietzel, E.; Maisner, A. Characterization of African bat henipavirus GH-M74a glycoproteins. J. Gen. Virol. 2014, 95, 539-548. [CrossRef]

39. Rissanen, I.; Ahmed, A.A.; Azarm, K.; Beaty, S.; Hong, P.; Nambulli, S.; Duprex, W.P.; Lee, B.; Bowden, T.A. Idiosyncratic Mòjiāng virus attachment glycoprotein directs a host-cell entry pathway distinct from genetically related henipaviruses. Nat. Commun. 2017, 8, 16060. [CrossRef] [PubMed]

40. Halpin, K.; Daszak, P.; Field, H.E.; Hyatt, A.D.; Smith, C.; Epstein, J.H.; Middleton, D.; Fogarty, R.; Hughes, T.; Bingham, J.; et al. Pteropid bats are confirmed as the reservoir hosts of henipaviruses: A comprehensive experimental study of virus transmission. Am. J. Trop. Med. Hyg. 2011, 85, 946-951. [CrossRef]

41. Yob, J.M.; Field, H.; Rashdi, A.M.; Morrissy, C.; Van Der Heide, B.; Rota, P.; Bin Adzhar, A.; White, J.; Daniels, P.; Jamaluddin, A.; et al. Nipah virus infection in bats (Order Chiroptera) in Peninsular Malaysia. Emerg. Infect. Dis. 2001, 7, 439-441. [CrossRef] [PubMed]

42. Chan, Y.-P.; Yan, L.; Feng, Y.-R.; Broder, C.C. Preparation of recombinant viral glycoproteins for novel and therapeutic antibody discovery. In Methods in Molecular Biology; Metzler, J.B., Ed.; Humana Press: Totowa, NJ, USA, 2009; Volume 525, pp. 31-58.

43. Harbury, P.B.; Zhang, T.; Kim, P.S.; Alber, T. A switch between two-, three-, and four-stranded coiled coils in GCN4 leucine zipper mutants. Science 1993, 262, 1401-1407. [CrossRef]

44. Xu, K.; Chan, Y.-P.; Bradel-Tretheway, B.; Akyol-Ataman, Z.; Zhu, Y.; Dutta, S.; Yan, L.; Feng, Y.; Wang, L.-F.; Skiniotis, G.; et al. Crystal structure of the pre-fusion Nipah virus fusion glycoprotein reveals a novel hexamer-of-trimers assembly. PLoS Pathog. 2015, 11, e1005322. [CrossRef] 
45. Chan, Y.-P.; Lu, M.; Dutta, S.; Yan, L.; Barr, J.; Flora, M.; Feng, Y.-R.; Xu, K.; Nikolov, D.B.; Wang, L.-F.; et al. Biochemical, conformational, and immunogenic analysis of soluble trimeric forms of Henipavirus fusion glycoproteins. J. Virol. 2012, 86, 11457-11471. [CrossRef] [PubMed]

46. Hall, M.P.; Unch, J.; Binkowski, B.F.; Valley, M.P.; Butler, B.L.; Wood, M.G.; Otto, P.; Zimmerman, K.; Vidugiris, G.; Machleidt, T.; et al. Engineered luciferase reporter from a deep sea shrimp utilizing a novel imidazopyrazinone substrate. ACS Chem. Biol. 2012, 7, 1848-1857. [CrossRef] [PubMed]

47. England, C.G.; Ehlerding, E.B.; Cai, W. NanoLuc: A small luciferase is brightening up the field of bioluminescence. Bioconjug. Chem. 2016, 27, 1175-1187. [CrossRef]

48. Dang, H.V.; Chan, Y.-P.; Park, Y.-J.; Snijder, J.; Da Silva, S.C.; Vu, B.; Yan, L.; Feng, Y.-R.; Rockx, B.; Geisbert, T.W.; et al. An antibody against the F glycoprotein inhibits Nipah and Hendra virus infections. Nat. Struct. Mol. Biol. 2019, 26, 980-987. [CrossRef]

49. Ohi, M.; Li, Y.; Cheng, Y.; Walz, T. Negative staining and image classification-powerful tools in modern electron microscopy. Biol. Proced. Online 2004, 6, 23-34. [CrossRef] [PubMed]

50. Sievers, F.; Wilm, A.; Dineen, D.; Gibson, T.J.; Karplus, K.; Li, W.; Lopez, R.; McWilliam, H.; Remmert, M.; Söding, J.; et al. Fast, scalable generation of high-quality protein multiple sequence alignments using Clustal Omega. Mol. Syst. Biol. 2011, 7, 539. [CrossRef]

51. Bertoni, M.; Kiefer, F.; Biasini, M.; Bordoli, L.; Schwede, T. Modeling protein quaternary structure of homo- and hetero-oligomers beyond binary interactions by homology. Sci. Rep. 2017, 7, 10480. [CrossRef] [PubMed]

52. Studer, G.; Rempfer, C.; Waterhouse, A.M.; Gumienny, R.; Haas, J.; Schwede, T. QMEANDisCo-distance constraints applied on model quality estimation. Bioinformatics 2020, 36, 1765-1771. [CrossRef] [PubMed]

53. Guex, N.; Peitsch, M.C.; Schwede, T. Automated comparative protein structure modeling with SWISS-MODEL and SwissPdbViewer: A historical perspective. Electrophoresis 2009, 30 (Suppl. 1), S162-S173. [CrossRef]

54. Bienert, S.; Waterhouse, A.; De Beer, T.A.P.; Tauriello, G.; Studer, G.; Bordoli, L.; Schwede, T. The SWISS-MODEL Repository-new features and functionality. Nucleic Acids Res. 2017, 45, D313-D319. [CrossRef] [PubMed]

55. Waterhouse, A.; Bertoni, M.; Bienert, S.; Studer, G.; Tauriello, G.; Gumienny, R.; Heer, F.T.; de Beer, T.A.P.; Rempfer, C.; Bordoli, L.; et al. SWISS-MODEL: Homology modelling of protein structures and complexes. Nucleic Acids Res. 2018, 46, W296-W303. [CrossRef] [PubMed]

56. Adamczak, R.; Porollo, A.; Meller, J. Combining prediction of secondary structure and solvent accessibility in proteins. Proteins 2005, 59, 467-475. [CrossRef] [PubMed]

57. Wagner, M.; Adamczak, R.; Porollo, A.A.; Meller, J. Linear regression models for solvent accessibility prediction in proteins. J. Comput. Biol. 2005, 12, 355-369. [CrossRef]

58. Adamczak, R.; Porollo, A.; Meller, J. Accurate prediction of solvent accessibility using neural networks-based regression. Proteins 2004, 56, 753-767. [CrossRef] [PubMed]

59. Ceroni, A.; Passerini, A.; Vullo, A.; Frasconi, P. DISULFIND: A disulfide bonding state and cysteine connectivity prediction server. Nucleic Acids Res. 2006, 34, W177-W181. [CrossRef]

60. Bossart, K.N.; Wang, L.-F.; Flora, M.N.; Chua, K.B.; Lam, S.K.; Eaton, B.T.; Broder, C.C. Membrane fusion tropism and heterotypic functional activities of the Nipah virus and Hendra virus envelope glycoproteins. J. Virol. 2002, 76, 11186-11198. [CrossRef] [PubMed]

61. Bossart, K.N.; Wang, L.-F.; Eaton, B.T.; Broder, C.C. Functional expression and membrane fusion tropism of the envelope glycoproteins of Hendra virus. Virology 2001, 290, 121-135. [CrossRef]

62. Pernet, O.; Beaty, S.; Lee, B. Functional rectification of the newly described African Henipavirus fusion glycoprotein (Gh-M74a). J. Virol. 2014, 88, 5171-5176. [CrossRef]

63. Cifuentes-Muñoz, N.; Sun, W.; Ray, G.; Schmitt, P.T.; Webb, S.; Gibson, K.; Dutch, R.E.; Schmitt, A.P. Mutations in the transmembrane domain and cytoplasmic tail of hendra virus fusion protein disrupt virus-like-particle assembly. J. Virol. 2017, 91, e00152-17. [CrossRef]

64. Westbury, H.A.; Hooper, P.T.; Selleck, P.W.; Murray, P.K. Equine morbillivirus pneumonia: Susceptibility of laboratory animals to the virus. Aust. Vet. J. 1995, 72, 278-279. [CrossRef]

65. Wong, K.T.; Grosjean, I.; Brisson, C.; Blanquier, B.; Fevre-Montange, M.; Bernard, A.; Loth, P.; Georges-Courbot, M.-C.; Chevallier, M.; Akaoka, H.; et al. A golden hamster model for human acute Nipah virus infection. Am. J. Pathol. 2003, 163, 2127-2137. [CrossRef]

66. Dups, J.; Middleton, D.; Yamada, M.; Monaghan, P.; Long, F.; Robinson, R.; Marsh, G.A.; Wang, L.-F. A new model for Hendra virus encephalitis in the mouse. PLoS ONE 2012, 7, e40308. [CrossRef] [PubMed]

67. Rockx, B. Recent developments in experimental animal models of Henipavirus infection. Pathog. Dis. 2014, 71, 199-206. [CrossRef] [PubMed]

68. Satterfield, B.A.; Cross, R.W.; Fenton, K.A.; Agans, K.N.; Basler, C.F.; Geisbert, T.W.; Mire, C.E. The immunomodulating V and W proteins of Nipah virus determine disease course. Nat. Commun. 2015, 6, 7483. [CrossRef]

69. Schountz, T.; Campbell, C.; Wagner, K.; Rovnak, J.; Martellaro, C.; DeBuysscher, B.L.; Feldmann, H.; Prescott, J. Differential innate immune responses elicited by Nipah virus and Cedar virus correlate with disparate in vivo pathogenesis in hamsters. Viruses 2019, 11, 291. [CrossRef] 